University of South Carolina

Scholar Commons

$4-11-2000$

\title{
An Optimal-Order Error Estimate for an ELLAM Scheme for Two- Dimensional Linear Advection-Diffusion Equations
}

Hong Wang

University of South Carolina - Columbia, wang@math.sc.edu

Follow this and additional works at: https://scholarcommons.sc.edu/math_facpub

Part of the Mathematics Commons

Publication Info

Published in Siam Journal of Numerical Analysis, Volume 37, Issue 4, 2000, pages 1338-1368.

(C) SIAM Journal On Numerical Analysis 2000, Society for Industrial and Applied Mathematics

Wang, H. (2000). An Optimal-Order Error Estimate for an ELLAM Scheme for Two-Dimensional Linear Advection-Diffusion Equations. SIAM Journal On Numerical Analysis, 37(4), 1338-1368. doi: 10.1137/ s0036142998335686

This Article is brought to you by the Mathematics, Department of at Scholar Commons. It has been accepted for inclusion in Faculty Publications by an authorized administrator of Scholar Commons. For more information, please contact digres@mailbox.sc.edu. 


\title{
AN OPTIMAL-ORDER ERROR ESTIMATE FOR AN ELLAM SCHEME FOR TWO-DIMENSIONAL LINEAR ADVECTION-DIFFUSION EQUATIONS*
}

\author{
HONG WANG ${ }^{\dagger}$
}

\begin{abstract}
An Eulerian-Lagrangian localized adjoint method (ELLAM) is presented and analyzed for two-dimensional linear advection-diffusion partial differential equations (PDEs). An optimal-order error estimate in the $L^{2}$ norm and a superconvergence estimate in a discrete $H^{1}$ norm are derived. Numerical experiments are performed to verify the theoretical estimates.
\end{abstract}

Key words. characteristic methods, convergence analysis, error estimates, Eulerian-Lagrangian methods, numerical solutions of advection-diffusion equations

AMS subject classifications. 65M12, 65M25, 65M60, 76M10, 76S05

PII. S0036142998335686

1. Introduction. Advection-diffusion partial differential equations (PDEs) describe miscible displacement flow processes in petroleum reservoir simulation, subsurface contaminant transport and remediation, disposal of nuclear waste in underground repositories, and many other applications [3, 13, 22]. These equations are characterized by a nondissipative (hyperbolic) advective transport component and a dissipative (parabolic) diffusive component, and are coupled to other equations (e.g., pressure equations in hydrosciences/petroleum industry) in applications. Their solutions typically have moving steep fronts that need to be resolved accurately. Standard finite difference or Galerkin finite element methods tend to yield numerical solutions with severe nonphysical oscillations. The classical upwind finite difference method greatly reduces these oscillations but introduces excessive numerical dispersion. Extensive research has been carried out to develop numerical methods that can overcome these difficulties and allow accurate numerical solutions with reasonable computational effort. Most Eulerian methods have been based on upstream weighting techniques. The optimal test function methods [2, 7] attempt to minimize the error in approximating spatial derivatives and yield an upstream bias in the resulting schemes. The methods in $[4,33]$ attempt to reduce the overall truncation error by using a nonzero spatial error to cancel the temporal error. The streamline diffusion finite element method adds a numerical diffusion only in the direction of streamlines with no cross-wind diffusion introduced [5, 17, 18]. High resolution methods, such as the total variation diminishing methods and the essentially nonoscillatory methods, are well suited for nonlinear hyperbolic conservation laws and resolve shock discontinuities in the solutions without excessive smearing or spurious oscillations $[9,10,12,15,24,25,27]$. By utilizing the hyperbolic transport behavior of advection-diffusion PDEs, characteristic methods discretize the temporal and advective terms through a characteristic tracking to significantly reduce the truncation errors $[19,20,21,28]$. Traditional forward or particle tracking methods advance the grids following the characteristics and greatly reduce temporal errors.

* Received by the editors March 18, 1998; accepted for publication (in revised form) September 3, 1999; published electronically April 11, 2000. This research was supported in part by grants DEF05-95ER25266 and ONR N00014-94-1-1163.

http://www.siam.org/journals/sinum/37-4/33568.html

†Department of Mathematics, University of South Carolina, Columbia, SC 29208 (hwang@ math.sc.edu). 
However, these methods often severely distort the evolving grids and greatly complicate the solution procedures. The modified method of characteristics (MMOC) [11] follows the flow by tracking the characteristics backward from a fixed grid at the current time step and avoids the grid distortion problems present in forward tracking methods. Moreover, the MMOC symmetrizes and stabilizes the governing PDEs, greatly reduces temporal truncation errors and allows for large time steps in a simulation without loss of accuracy, and eliminates the excessive numerical dispersion and grid orientation effects present in many Eulerian methods [13, 14]. However, many characteristic methods fail to conserve mass and have difficulties in treating general flux boundary conditions when a characteristic intersects the boundary of the domain.

The Eulerian-Lagrangian localized adjoint method (ELLAM) was presented in [6, 16] for solving one-dimensional (constant-coefficient) advection-diffusion PDEs. The ELLAM formalism provides a general characteristic solution procedure for advectiondominated PDEs and a consistent framework for treating general boundary conditions and maintaining mass conservation. Thus, the ELLAM methodology overcomes the principal shortcomings of many characteristic methods while maintaining their numerical advantages. We previously conducted numerical experiments in [30, 31], which showed that the ELLAM methods often outperform many well received and widely used methods. A different but related method to the ELLAM is the characteristic mixed finite element method [1,34], which uses piecewise-constant spacetime test functions. As with the standard mixed finite element method, a coupled system results for both the concentration and the diffusive flux. The theoretically proven error estimate is $\mathcal{O}\left(h^{\frac{3}{2}}\right)$ for grid size $h$, which is suboptimal by a factor $\mathcal{O}\left(h^{\frac{1}{2}}\right)$.

ELLAM methods introduce further difficulties and complexities to the already complicated analyses of characteristic methods. When a characteristic intersects the boundary of the domain, the uniform time step used in the MMOC artificially increases the time period of the physical diffusion and thus introduces numerical diffusion into its formulation. Nevertheless, this uniform time step guarantees a uniform coercivity of the MMOC scheme on the Sobolev space $H^{1}(\Omega)$, which is essential in its theoretical analysis [11]. In contrast, the ELLAM schemes introduce spatially dependent time steps to accurately measure the time period of physical diffusion and yield time step degeneracies near boundaries. Consequently, the techniques used in the analyses for MMOC can no longer be applied. We previously overcame these difficulties and derived optimal-order $L^{2}$ convergence and superconvergence estimates for the ELLAM schemes for one-dimensional advection-diffusion PDEs [29, 32]. But these analyses are based on a generalized Sobolev inequality that we proved, which in turn depends on the Sobolev embedding theorem $H^{1}(\Omega) \hookrightarrow C(\bar{\Omega})$ that is true only in one space dimension. Hence, the analyses would not carry over to multidimensional problems. In this paper we adopt a different approach to derive an optimal-order $L^{2}$ error estimate and a superconvergence estimate for the ELLAM schemes for twodimensional advection-diffusion PDEs.

This paper is organized as follows. In section 2 we present an ELLAM scheme. Section 3 cites known results that are used in this paper. In section 4 we prove an optimal-order $L^{2}$ error estimate and a superconvergence estimate for the derived ELLAM scheme. In section 5 and section 6 we prove auxiliary lemmas used in the proof in section 4 . In section 7 we carry out numerical experiments to verify the theoretical error estimates. 
2. An ELLAM scheme. We consider the following linear advection-diffusion PDE

$$
c_{t}+\nabla \cdot(\mathbf{v}(\mathbf{x}, t) c-D(\mathbf{x}, t) \nabla c)=f(\mathbf{x}, t), \quad \mathbf{x}=(x, y) \in \Omega, \quad t \in[0, T] .
$$

Here $\Omega=\left(x^{L}, x^{R}\right) \times\left(y^{L}, y^{R}\right)$ is a two-dimensional rectangular domain with the boundary $\Gamma:=\partial \Omega . \quad c_{t}=\frac{\partial c}{\partial t}, \nabla c=\left(\frac{\partial c}{\partial x}, \frac{\partial c}{\partial y}\right) . \quad \mathbf{v}(\mathbf{x}, t)=\left(V^{(1)}(\mathbf{x}, t), V^{(2)}(\mathbf{x}, t)\right)$ is a fluid velocity field, $D(\mathbf{x}, t)$ is a diffusion coefficient, $f(\mathbf{x}, t)$ is a given function, and $c(\mathbf{x}, t)$ is the solute concentration of a dissolved substance. Let $\Gamma^{(I)}$ and $\Gamma^{(O)}$ be the inflow and outflow boundaries identified by

$$
\begin{aligned}
\Gamma^{(I)} & :=\{\mathbf{x} \mid \mathbf{x} \in \Gamma, \mathbf{v} \cdot \boldsymbol{\nu}<0\}, \\
\Gamma^{(O)} & :=\{\mathbf{x} \mid \mathbf{x} \in \Gamma, \mathbf{v} \cdot \boldsymbol{\nu}>0\},
\end{aligned}
$$

with $\boldsymbol{\nu}(\mathbf{x})$ being the unit outward normal. The ELLAM scheme presented in this article can treat any boundary conditions [6, 23, 31]. We previously derived optimalorder error estimates for a family of ELLAM schemes for one-dimensional advectiondiffusion PDEs with all combinations of inflow and outflow Dirichlet, Neumann, and flux boundary conditions $[29,32]$. For simplicity of exposition, we consider the following initial and boundary conditions:

$$
\begin{aligned}
& (\mathbf{v} c-D \nabla c) \cdot \boldsymbol{\nu}=g^{(I)}(\mathbf{x}, t), \quad \mathbf{x} \in \Gamma^{(I)}, \quad t \in[0, T], \\
& c=g^{(O)}(\mathbf{x}, t), \quad \mathbf{x} \in \Gamma^{(O)}, \quad t \in[0, T], \\
& c(\mathbf{x}, 0)=c_{0}(\mathbf{x}), \quad \mathbf{x} \in \Omega,
\end{aligned}
$$

where $\frac{\partial c}{\partial \nu}:=\nabla c \cdot \nu, g^{(I)}(\mathbf{x}, t), g^{(O)}(\mathbf{x}, t)$, and $c_{0}(\mathbf{x})$ are prescribed functions.

2.1. Characteristic tracking and test functions. Let $I, J$, and $N$ be positive integers. We define a uniform space-time partition on $\Omega \times[0, T]=\left(x^{L}, x^{R}\right) \times\left(y^{L}, y^{R}\right) \times$ $[0, T]$ by

$$
\begin{array}{llll}
x_{i}:=x^{L}+i \Delta x, & i=0,1, \ldots, I, & \Delta x:=\frac{x^{R}-x^{L}}{I} \\
y_{j}:=y^{L}+j \Delta y, & j=0,1, \ldots, J, & \Delta y:=\frac{y^{R}-y^{L}}{J} \\
t_{n}:=n \Delta t, & n=0,1, \ldots, N, & \Delta t:=\frac{T}{N} .
\end{array}
$$

The partition is assumed to be quasi-uniform

$$
0<h:=\max \{\Delta x, \Delta y\} \leq M_{0} \min \{\Delta x, \Delta y\} .
$$

Let $\phi_{i, j}(\mathbf{x})(0 \leq i \leq I, 0 \leq j \leq J)$ be the standard piecewise-bilinear basis functions associated with the node $\mathbf{x}_{i, j}=\left(x_{i}, y_{j}\right)$ (i.e, $\phi_{i, j}\left(\mathbf{x}_{k, l}\right)=\delta_{i, k} \delta_{j, l}$, where $\delta_{i, k}=1$ if $i=k$ or 0 otherwise). Let

$$
\mathcal{S}_{h}(\Omega):=\operatorname{span}\left\{\phi_{i, j}\right\}_{\{0 \leq i \leq I, 0 \leq j \leq J\}} \bigcap\left\{\left.\phi_{i, j}\right|_{\Gamma^{(O)}}=0\right\} .
$$

In the ELLAM framework $[6,23,31]$ the test functions $w$ are chosen to satisfy the adjoint equation of the hyperbolic part of (2.1)

$$
w_{t}+\mathbf{v} \cdot \nabla w=0
$$


This implies that the test functions $w$ should be constant along the characteristics defined by the ordinary differential equation

$$
\frac{d \mathbf{y}}{d t}=\mathbf{v}(\mathbf{y}, t)
$$

Because (2.8) does not have closed-form analytical solutions in general, we track the characteristics approximately by the Euler method. For any $\mathbf{x} \in \Omega$ we define an approximate characteristic $\mathbf{y}=\mathbf{r}\left(\theta ; \mathbf{x}, t_{n}\right)$ emanating backward from $\mathbf{x}$ at time $t_{n}$ by

$$
\mathbf{r}\left(\theta ; \mathbf{x}, t_{n}\right):=\mathbf{x}-\mathbf{v}\left(\mathbf{x}, t_{n}\right)\left(t_{n}-\theta\right), \quad \theta \in\left[t^{*}(\mathbf{x}), t_{n}\right]
$$

where $t^{*}(\mathbf{x}):=t_{n-1}$ if $\mathbf{r}\left(\theta ; \mathbf{x}, t_{n}\right)$ does not backtrack to the boundary $\Gamma$ for $\theta \in$ $\left[t_{n-1}, t_{n}\right]$, or $t^{*}(\mathbf{x}) \in\left[t_{n-1}, t_{n}\right]$ is the time instant when $\mathbf{r}\left(\theta ; \mathbf{x}, t_{n}\right)$ intersects the boundary $\Gamma$ (i.e., $\mathbf{r}\left(t^{*}(\mathbf{x}) ; \mathbf{x}, t_{n}\right) \in \Gamma$ ) otherwise. For any $(\mathbf{x}, t) \in \Gamma^{(O)} \times\left[t_{n-1}, t_{n}\right]$, the approximate characteristic $\mathbf{y}=\mathbf{r}(\theta ; \mathbf{x}, t)$ extending from $(\mathbf{x}, t)$ is given by

$$
\mathbf{r}(\theta ; \mathbf{x}, t):=\mathbf{x}-\mathbf{v}(\mathbf{x}, t)(t-\theta), \quad \theta \in\left[t^{*}(\mathbf{x}, t), t\right],
$$

where $t^{*}(\mathbf{x}, t):=t_{n-1}$ if $\mathbf{r}(\theta ; \mathbf{x}, t)$ does not backtrack to the boundary $\Gamma$ for $\theta \in$ $\left[t_{n-1}, t\right]$, or $t^{*}(\mathbf{x}, t) \in\left[t_{n-1}, t\right]$ is the time instant when $\mathbf{r}(\theta ; \mathbf{x}, t)$ intersects the boundary $\Gamma$ (i.e., $\left.\mathbf{r}\left(t^{*}(\mathbf{x}, t) ; \mathbf{x}, t\right) \in \Gamma\right)$ otherwise.

To accurately measure the effect of physical diffusion on a particle traveling from $\Omega$ at time $t_{n-1}$ or the inflow boundary to $\Omega$ at the current time $t_{n}$, in addition to the time step $\Delta t$ defined in (2.4) we define a spatially dependent time step

$$
\Delta t(\mathbf{x}):=t_{n}-t^{*}(\mathbf{x})
$$

For any function $\phi(\mathbf{x}) \in \mathcal{S}_{h}$, we define $w(\mathbf{x}, t)$ to satisfy the homogeneous Dirichlet boundary condition at the outflow boundary and to be a constant extension of $\phi(\mathbf{x})$ into the space-time strip $\bar{\Omega} \times\left[t_{n-1}, t_{n}\right]$ along the approximate characteristics

$$
\begin{aligned}
w\left(\mathbf{r}\left(\theta ; \mathbf{x}, t_{n}\right), \theta\right) & :=w\left(\mathbf{x}, t_{n}\right), & & \theta \in\left[t^{*}(\mathbf{x}), t_{n}\right], \mathbf{x} \in \bar{\Omega} \\
w(\mathbf{r}(\theta ; \mathbf{x}, t), \theta) & :=w(\mathbf{x}, t), & & \theta \in\left[t^{*}(\mathbf{x}, t), t\right] \\
w(\mathbf{x}, t) & :=0, & & (\mathbf{x}, t) \in \Gamma^{(O)} \times\left[t_{n-1}, t_{n}\right] .
\end{aligned}
$$

Multiplying (2.1) by any of such test functions we obtain a space-time weak formulation for (2.1)

$$
\begin{aligned}
\int_{\Omega} & c\left(\mathbf{x}, t_{n}\right) w\left(\mathbf{x}, t_{n}\right) d \mathbf{x}+\int_{t_{n-1}}^{t_{n}} \int_{\Omega}(\nabla w \cdot D \nabla c)(\mathbf{z}, \theta) d \mathbf{z} d \theta \\
& +\int_{t_{n-1}}^{t_{n}} \int_{\Gamma}(\mathbf{v} c-D \nabla c)(\mathbf{x}, t) \cdot \boldsymbol{\nu}(\mathbf{x}) w(\mathbf{x}, t) d s d t \\
& -\int_{t_{n-1}}^{t_{n}} \int_{\Omega} c(\mathbf{z}, \theta)\left(w_{\theta}+\mathbf{v} \cdot \nabla w\right)(\mathbf{z}, \theta) d \mathbf{z} d \theta \\
= & \int_{\Omega} c\left(\mathbf{x}, t_{n-1}\right) w\left(\mathbf{x}, t_{n-1}^{+}\right) d \mathbf{x}+\int_{t_{n-1}}^{t_{n}} \int_{\Omega} f(\mathbf{z}, \theta) w(\mathbf{z}, \theta) d \mathbf{z} d \theta
\end{aligned}
$$

where $w\left(\mathbf{x}, t_{n-1}^{+}\right):=\lim _{t \rightarrow t_{n-1}^{+}} w(\mathbf{x}, t)$ to take into account the fact that $w(\mathbf{x}, t)$ is discontinuous in time at time $t_{n-1}$. 
2.2. Evaluation of the source and the diffusion terms. We interchange the order of integration in the second (source) term on the right-hand side of $(2.13)$ and apply the Euler quadrature to the temporal integral at time $t_{n}$, yielding

$$
\begin{aligned}
& \int_{t_{n-1}}^{t_{n}} \int_{\Omega} f(\mathbf{y}, \theta) w(\mathbf{y}, \theta) d \mathbf{y} d \theta \\
& \quad=\int_{\Omega} \int_{t^{*}(\mathbf{x})}^{t_{n}} f\left(\mathbf{r}\left(\theta ; \mathbf{x}, t_{n}\right), \theta\right) w\left(\mathbf{x}, t_{n}\right)\left|\mathbf{J}\left(\theta ; \mathbf{x}, t_{n}\right)\right| d \theta d \mathbf{x} \\
& =\int_{\Omega} \Delta t(\mathbf{x}) f\left(\mathbf{x}, t_{n}\right) w\left(\mathbf{x}, t_{n}\right) d \mathbf{x}+E_{1}(f, w)
\end{aligned}
$$

where

$$
\left|\mathbf{J}\left(\theta ; \mathbf{x}, t_{n}\right)\right|:=\left|\frac{\partial \mathbf{r}\left(\theta ; \mathbf{x}, t_{n}\right)}{\partial \mathbf{x}}\right|=1+\mathcal{O}\left(t_{n}-\theta\right)
$$

is the Jacobian of the transformation from $\mathbf{x}$ to $\mathbf{r}\left(\theta ; \mathbf{x}, t_{n}\right)$, and $E_{1}(f, w)$ is the truncation error defined by

$$
\begin{aligned}
& E_{1}(f, w) \\
& \quad:=\int_{\Omega}\left\{\int_{t^{*}(\mathbf{x})}^{t_{n}}\left[f\left(\mathbf{r}\left(\theta ; \mathbf{x}, t_{n}\right), \theta\right)\left|\mathbf{J}\left(\theta ; \mathbf{x}, t_{n}\right)\right|-f\left(\mathbf{x}, t_{n}\right)\right] d \theta\right\} w\left(\mathbf{x}, t_{n}\right) d \mathbf{x} .
\end{aligned}
$$

We similarly evaluate the second (diffusion) term on the left-hand side of (2.13) to get

$$
\begin{aligned}
& \int_{t_{n-1}}^{t_{n}} \int_{\Omega} \nabla w(\mathbf{y}, \theta) \cdot(D \nabla c)(\mathbf{y}, \theta) d \mathbf{y} d \theta \\
& \quad=\int_{\Omega} \int_{t^{*}(\mathbf{x})}^{t_{n}} \nabla w\left(\mathbf{r}\left(\theta ; \mathbf{x}, t_{n}\right), \theta\right) \cdot(D \nabla c)\left(\mathbf{r}\left(\theta ; \mathbf{x}, t_{n}\right), \theta\right)\left|\mathbf{J}\left(\theta ; \mathbf{x}, t_{n}\right)\right| d \theta d \mathbf{x} \\
& \quad=\int_{\Omega} \Delta t(\mathbf{x}) D\left(\mathbf{x}, t_{n}\right) \nabla w\left(\mathbf{x}, t_{n}\right) \cdot \nabla c\left(\mathbf{x}, t_{n}\right) d \mathbf{x}+E_{2}(D, c, w)
\end{aligned}
$$

where $E_{2}(D, c, w)$ is the truncation error term given by

$$
\begin{aligned}
E_{2}(D, c, w):=\int_{\Omega} & \left\{\int _ { t ^ { * } ( \mathbf { x } ) } ^ { t _ { n } } \left[(\nabla w \cdot D \nabla c)\left(\mathbf{r}\left(\theta ; \mathbf{x}, t_{n}\right), \theta\right)\left|\mathbf{J}\left(\theta ; \mathbf{x}, t_{n}\right)\right|\right.\right. \\
& \left.\left.-(\nabla w \cdot D \nabla c)\left(\mathbf{x}, t_{n}\right)\right] d \theta\right\} d \mathbf{x} .
\end{aligned}
$$

2.3. An ELLAM scheme. Substituting (2.14) and (2.17) into (2.13) and incorporating the boundary conditions (2.3), we obtain a reference equation for $(2.1)$ with the boundary conditions $(2.3)$

$$
\begin{gathered}
\int_{\Omega} c\left(\mathbf{x}, t_{n}\right) w\left(\mathbf{x}, t_{n}\right) d \mathbf{x}+\int_{\Omega} \Delta t(\mathbf{x}) D\left(\mathbf{x}, t_{n}\right) \nabla w\left(\mathbf{x}, t_{n}\right) \cdot \nabla c\left(\mathbf{x}, t_{n}\right) d \mathbf{x} \\
=\int_{\Omega} c\left(\mathbf{x}, t_{n-1}\right) w\left(\mathbf{x}, t_{n-1}^{+}\right) d \mathbf{x}+\int_{\Omega} \Delta t(\mathbf{x}) f\left(\mathbf{x}, t_{n}\right) w\left(\mathbf{x}, t_{n}\right) d \mathbf{x} \\
\quad-\int_{t_{n-1}}^{t_{n}} \int_{\Gamma^{(I)}} g^{(I)}(\mathbf{x}, t) w(\mathbf{x}, t) d s d t+E(D, f, c, w),
\end{gathered}
$$


where $E(D, f, c, w)$ is the truncation error defined by

$$
\begin{aligned}
& E(D, f, c, w) \\
& \quad:=\int_{t_{n-1}}^{t_{n}} \int_{\Omega} c(\mathbf{z}, \theta)\left(w_{\theta}+\mathbf{v} \cdot \nabla w\right)(\mathbf{z}, \theta) d \mathbf{z} d \theta+E_{1}(f, w)-E_{2}(D, c, w)
\end{aligned}
$$

with $E_{1}(f, w)$ and $E_{2}(D, c, w)$ being given by (2.16) and (2.18), respectively.

With the solution $c\left(\mathbf{x}, t_{n-1}\right)$ on $\Omega$ at time $t_{n-1}$ and the boundary conditions (2.3), the reference equation (2.19) determines the unknown solution $c\left(\mathbf{x}, t_{n}\right)$ for $\mathbf{x} \in \Omega$ at time $t_{n}$. We define the trial functions $C\left(\mathbf{x}, t_{n}\right)$ to be of the form

$$
\begin{aligned}
C\left(\mathbf{x}, t_{n}\right):=\sum_{\substack{\mathbf{x}_{i, j} \in \bar{\Omega} \backslash \Gamma^{(O)} \\
\mathbf{x} \in \bar{\Omega}, \quad n=0,1, \ldots, N .}} C\left(\mathbf{x}_{i, j}, t_{n}\right) \phi_{i, j}(\mathbf{x})+\sum_{\mathbf{x}_{i, j} \in \Gamma^{(O)}} g^{(O)}\left(\mathbf{x}_{i, j}, t_{n}\right) \phi_{i, j}(\mathbf{x}), \\
\end{aligned}
$$

After we define the trial and test functions, we now present an ELLAM scheme based on the reference equation (2.19): Find solution $C$, which is of the form $(2.21)$ for $\mathbf{x} \in \bar{\Omega}$ at time $t_{n}$ such that

$$
\begin{aligned}
\int_{\Omega} C & \left(\mathbf{x}, t_{n}\right) w\left(\mathbf{x}, t_{n}\right) d \mathbf{x}+\int_{\Omega} \Delta t(\mathbf{x}) D\left(\mathbf{x}, t_{n}\right) \nabla w\left(\mathbf{x}, t_{n}\right) \cdot \nabla C\left(\mathbf{x}, t_{n}\right) d \mathbf{x} \\
= & \int_{\Omega} C\left(\mathbf{x}, t_{n-1}\right) w\left(\mathbf{x}, t_{n-1}^{+}\right) d \mathbf{x}+\int_{\Omega} \Delta t(\mathbf{x}) f\left(\mathbf{x}, t_{n}\right) w\left(\mathbf{x}, t_{n}\right) d \mathbf{x} \\
& -\int_{t_{n-1}}^{t_{n}} \int_{\Gamma^{(I)}} g^{(I)}(\mathbf{x}, t) w(\mathbf{x}, t) d s d t
\end{aligned}
$$

holds for any test functions defined in section 2.1.

Remark 2.1. The ELLAM scheme (2.22) symmetrizes the governing PDE (2.1), and generates a symmetric and positive-definite, sparse coefficient matrix with an improved condition number of order $\mathcal{O}\left(1+\frac{D \Delta t}{h^{2}}\right)$. Second, the ELLAM scheme eliminates the majority of the temporal errors, and allows large time steps to be used without loss of accuracy. Third, the ELLAM scheme naturally incorporates the boundary conditions (2.3) into its formulation. We refer interested readers to [31] for details on implementational issues. Finally, our earlier numerical experiments showed that the ELLAM scheme often outperforms many well received and widely used numerical methods $[30,31]$.

3. Preliminaries. In this section, we present preliminaries that are needed for the theoretical analysis in this paper.

3.1. Notions. Let $L^{p}(\Omega), 1 \leq p \leq+\infty$, be the standard normed spaces of $p$ th power Lebesgue integrable functions. Then we define the Sobolev spaces

$$
W^{m, p}(\Omega):=\left\{v(\mathbf{x}) \mid \frac{\partial^{i+j} v(x, y)}{\partial x^{i} \partial y^{j}} \in L^{p}(\Omega), \quad 0 \leq i+j \leq m\right\}
$$

with the (semi-) norms 


$$
\begin{aligned}
\|v\|_{W^{m, p}(\Omega)} & := \begin{cases}{\left[\sum_{0 \leq i+j \leq m}\left\|\frac{\partial^{i+j} v(x, y)}{\partial x^{i} \partial y^{j}}\right\|_{L^{p}(\Omega)}^{p},\right.} & 1 \leq p<+\infty, \\
\max _{0 \leq i+j \leq m}\left\|\frac{\partial^{i+j} v(x, y)}{\partial x^{i} \partial y^{j}}\right\|_{L^{\infty}(\Omega)}^{\frac{1}{p}}, & p=+\infty,\end{cases} \\
\|v\|_{\hat{H}^{1}(\Omega)} & :=\left[\sum_{i=1}^{I} \sum_{j=1}^{J} \mid \nabla v\left(\left.\mathbf{x}_{\left.i-\frac{1}{2}, j-\frac{1}{2}\right)}\right|^{2} h^{2}\right]^{\frac{1}{2}},\right.
\end{aligned}
$$

where $\mathbf{x}_{i-\frac{1}{2}, j-\frac{1}{2}}:=\left(x_{i-\frac{1}{2}}, y_{j-\frac{1}{2}}\right)$, with $x_{i-\frac{1}{2}}:=x_{i-1}+\frac{\Delta x}{2}$ and $y_{j-\frac{1}{2}}:=y_{j-1}+\frac{\Delta y}{2}$. In particular, we let $H^{m}(\Omega):=W^{m, 2}(\Omega), H^{0}(\Omega):=L^{2}(\Omega)$, and $W^{0, p}(\Omega)=L^{p}(\Omega)$.

In addition, for $1 \leq p, q \leq+\infty$ we define the normed spaces

$$
\begin{aligned}
& L^{q}\left(t_{1}, t_{2} ; W^{m, p}(\Omega)\right):= \\
& \quad\left\{w(\mathbf{x}, t) \mid w(\cdot, t):\left(t_{1}, t_{2}\right) \longmapsto W^{m, p}(\Omega), \quad\|w(\cdot, t)\|_{W^{m, p}(\Omega)} \in L^{q}\left(t_{1}, t_{2}\right)\right\}
\end{aligned}
$$

with the norms

$$
\begin{aligned}
& \|w\|_{L^{q}\left(t_{1}, t_{2} ; W^{m, p}(\Omega)\right)}:=\|\| w(\cdot, t)\left\|_{W^{m, p}(\Omega)}\right\|_{L^{q}\left(t_{1}, t_{2}\right)}, \quad 1 \leq p, q \leq+\infty, \\
& \|w\|_{\hat{L}^{q}\left(0, T ; W^{m, p}(\Omega)\right)}:= \begin{cases}{\left[\sum_{n=0}^{N}\left\|w\left(\cdot, t_{n}\right)\right\|_{W^{m, p}(\Omega)}^{q} \Delta t\right]^{\frac{1}{q}},} & 1 \leq q<+\infty, \\
\max _{0 \leq n \leq N}\left\|w\left(\cdot, t_{n}\right)\right\|_{W^{m, p}(\Omega)}, & q=+\infty,\end{cases} \\
& \|w\|_{\hat{L}^{2}\left(0, T ; \hat{H}^{1}(\Omega)\right)}:=\left[\sum_{n=0}^{N} \sum_{i=1}^{I} \sum_{j=1}^{J}\left|\nabla w\left(\mathbf{x}_{i-\frac{1}{2}, j-\frac{1}{2}}, t_{n}\right)\right|^{2} h^{2} \Delta t\left(\mathbf{x}_{i-\frac{1}{2}, j-\frac{1}{2}}\right)\right]^{\frac{1}{2}}
\end{aligned}
$$

We also make the following assumptions on (2.1):

Assumption (A):

(i) $D(\mathbf{x}, t), f(\mathbf{x}, t) \in W^{1, \infty}(\Omega \times(0, T))$ and $\mathbf{v}(\mathbf{x}, t) \in\left(W^{1, \infty}(\Omega \times(0, T))\right)^{2}$.

(ii) There exist positive constants $D_{\min }$ and $D_{\max }$, such that

$$
0<D_{\min } \leq D(\mathbf{x}, t) \leq D_{\max }<+\infty \quad \forall(\mathbf{x}, t) \in \bar{\Omega} \times[0, T] .
$$

(iii) The solution $c(\mathbf{x}, t) \in L^{\infty}\left(0, T ; W^{2, \infty}(\Omega)\right)$ and $c_{t}(\mathbf{x}, t) \in L^{2}\left(0, T ; H^{2}(\Omega)\right)$.

3.2. Auxiliary functions. For any $t \in[0, T]$, we introduce the Ritz projection $\Pi c(\mathbf{x}, t)$ of $c(\mathbf{x}, t)$, which is of the form (2.21), such that [26]

$$
\int_{\Omega} D(\mathbf{x}, t) \nabla \phi(\mathbf{x}) \cdot \nabla(\Pi c(\mathbf{x}, t)-c(\mathbf{x}, t)) d \mathbf{x}=0 \quad \forall \phi(\mathbf{x}) \in \mathcal{S}_{h}(\Omega) .
$$

It is well known that the following estimates hold for $k=0,1[8,35]$ :

$$
\begin{array}{ll}
\|\Pi c(\cdot, t)-c(\cdot, t)\|_{H^{k}(\Omega)} & \leq M h^{2-k}\|c(\cdot, t)\|_{H^{2}(\Omega)}, \\
\left\|(\Pi c(\cdot, t)-c(\cdot, t))_{t}\right\|_{H^{k}(\Omega)} & \leq M h^{2-k}\left(\|c(\cdot, t)\|_{H^{2}(\Omega)}+\left\|c_{t}(\cdot, t)\right\|_{H^{2}(\Omega)}\right), \\
\|\Pi c(\cdot, t)-c(\cdot, t)\|_{W^{k, \infty}(\Omega)} & \leq M h^{2-k}|\ln h|^{1-k}\|c(\cdot, t)\|_{W^{2, \infty}(\Omega)},
\end{array}
$$




$$
\begin{array}{lll}
\|\nabla \phi\|_{L^{\infty}(\Omega)} & \leq M h^{-1}\|\nabla \phi\|_{L^{2}(\Omega)}, & \|\phi\|_{L^{\infty}(\Omega)} \leq M h^{-1}\|\phi\|_{L^{2}(\Omega)} \\
\|\nabla \phi\|_{L^{2}(\Omega)} & \leq M h^{-1}\|\phi\|_{L^{2}(\Omega)} & \forall \phi(\mathbf{x}) \in \mathcal{S}_{h}(\Omega) .
\end{array}
$$

Then we introduce the following notations:

$$
\begin{array}{lll}
e\left(\mathbf{x}, t_{n}\right) & :=C\left(\mathbf{x}, t_{n}\right)-c\left(\mathbf{x}, t_{n}\right), & \eta(\mathbf{x}, t):=\Pi c(\mathbf{x}, t)-c(\mathbf{x}, t), \\
\xi\left(\mathbf{x}, t_{n}\right) & :=C\left(\mathbf{x}, t_{n}\right)-\Pi c\left(\mathbf{x}, t_{n}\right), & \mathbf{x} \in \Omega, n=0,1, \ldots, N .
\end{array}
$$

Using the fact that $\xi\left(\mathbf{x}, t_{n}\right) \in \mathcal{S}_{h}(\Omega)$, we have

$$
\begin{aligned}
\xi\left(\mathbf{x}, t_{n}\right)= & \sum_{j=0}^{J} \sum_{i=0}^{I} \xi\left(\mathbf{x}_{i, j}, t_{n}\right) w_{i, j}\left(\mathbf{x}, t_{n}\right), \quad \mathbf{x} \in \Omega \\
\xi\left(\mathbf{r}\left(\theta ; \mathbf{x}, t_{n}\right), \theta\right)= & \sum_{j=0}^{J} \sum_{i=0}^{I} \xi\left(\mathbf{x}_{i, j}, t_{n}\right) w_{i, j}\left(\mathbf{r}\left(\theta ; \mathbf{x}, t_{n}\right), \theta\right), \\
& \theta \in\left[t^{*}(\mathbf{x}), t_{n}\right], \quad \mathbf{x} \in \bar{\Omega} .
\end{aligned}
$$

In this paper we use $\varepsilon$ to denote an arbitrary small number and $M$ to denote a generic constant, which may have different values at different places.

4. Error estimates. Because the physically relevant spatially dependent time step $\Delta t(\mathbf{x})$ defined in (2.11) degenerates near the boundary, the techniques used in the analyses for MMOC and other characteristic methods no longer apply since they rely heavily on a uniform $H^{1}(\Omega)$ coercivity of their formulations [11]. We previously proved an optimal-order $L^{2}$ error estimate and a superconvergence estimate for the ELLAM schemes for one-dimensional advection-diffusion PDEs [29, 32]. These analyses are based on a generalized Sobolev inequality that we derived, which in turn depends on the Sobolev embedding theorem $H^{1}(\Omega) \hookrightarrow C(\bar{\Omega})$ that is true only in one space dimension. Hence, the analyses would not carry over to multidimensional problems. In this section, we adopt a different approach to derive an optimal order error estimate for the ELLAM scheme (2.22).

Subtracting (2.22) from (2.19) and choosing the test function $w=\xi$ in the resulting equation, we obtain the following relation:

$$
\begin{aligned}
& \int_{\Omega} e\left(\mathbf{x}, t_{n}\right) \xi\left(\mathbf{x}, t_{n}\right) d \mathbf{x}+\int_{\Omega} \Delta t(\mathbf{x}) D\left(\mathbf{x}, t_{n}\right) \nabla \xi\left(\mathbf{x}, t_{n}\right) \cdot \nabla e\left(\mathbf{x}, t_{n}\right) d \mathbf{x} \\
& =\int_{\Omega} e\left(\mathbf{x}, t_{n-1}\right) \xi\left(\mathbf{x}, t_{n-1}^{+}\right) d \mathbf{x}-E(D, f, c, \xi) .
\end{aligned}
$$

We decompose the global truncation error $e=C-c$ as the sum $e=\xi+\eta$, in which the error estimates for $\eta$ are given in (3.7). Therefore, we only need to derive an estimate for $\xi$. Hence, we rewrite (4.1) in terms of $\xi$ and $\eta$

$$
\begin{aligned}
\int_{\Omega} & \xi^{2}\left(\mathbf{x}, t_{n}\right) d \mathbf{x}+\int_{\Omega} \Delta t(\mathbf{x}) D\left(\mathbf{x}, t_{n}\right)\left|\nabla \xi\left(\mathbf{x}, t_{n}\right)\right|^{2} d \mathbf{x} \\
= & \int_{\Omega} \xi\left(\mathbf{x}, t_{n-1}\right) \xi\left(\mathbf{x}, t_{n-1}^{+}\right) d \mathbf{x}+\int_{\Omega} \eta\left(\mathbf{x}, t_{n-1}\right) \xi\left(\mathbf{x}, t_{n-1}^{+}\right) d \mathbf{x} \\
& -\int_{\Omega} \eta\left(\mathbf{x}, t_{n}\right) \xi\left(\mathbf{x}, t_{n}\right) d \mathbf{x}-\int_{\Omega} \Delta t(\mathbf{x}) D\left(\mathbf{x}, t_{n}\right) \nabla \xi\left(\mathbf{x}, t_{n}\right) \cdot \nabla \eta\left(\mathbf{x}, t_{n}\right) d \mathbf{x} \\
& -E(D, f, c, \xi) .
\end{aligned}
$$


The terms on the left-hand side of (4.2) are in a desired form; we estimate those on its right-hand side. For any $\theta \in\left[t_{n-1}, t_{n}\right]$, let

$$
\begin{array}{lll}
\Omega^{(O)}(\theta) & :=\left\{\mathbf{y} \in \Omega \mid \exists(\mathbf{x}, t) \in \Gamma \times\left[\theta, t_{n}\right],\right. & \text { such that (s.t.) } \mathbf{y}=\mathbf{r}(\theta ; \mathbf{x}, t)\} \\
\Omega^{(I)}(\theta) & :=\left\{\mathbf{x} \in \Omega \mid \exists(\mathbf{y}, \gamma) \in \Gamma \times\left[t_{n-1}, \theta\right],\right. & \text { s.t. } \mathbf{y}=\mathbf{r}(\gamma ; \mathbf{x}, \theta)\}
\end{array}
$$

be the sets of points that will flow out of the domain $\Omega$ during the time period $\left[\theta, t_{n}\right]$ or that flew into $\Omega$ during the time period $\left[t_{n-1}, \theta\right]$. For any $\mathbf{x} \in \Omega \backslash \Omega^{(O)}\left(t_{n-1}\right)$ or $\mathbf{x} \in \Omega^{(O)}\left(t_{n-1}\right)$, we define $\tilde{\mathbf{x}} \in \overline{\Omega \backslash \Omega^{(I)}\left(t_{n}\right)}$ or $(\tilde{\mathbf{x}}, \tilde{t}) \in \Gamma^{(O)} \times\left[t_{n-1}, t_{n}\right]$ by

$$
\begin{aligned}
\mathbf{x}=\mathbf{r}\left(t_{n-1} ; \tilde{\mathbf{x}}, t_{n}\right)= & \dot{\tilde{\mathbf{x}}}-\mathbf{v}\left(\tilde{\mathbf{x}}, t_{n}\right) \Delta t \quad \forall \mathbf{x} \in \Omega \backslash \Omega^{(O)}\left(t_{n-1}\right) \\
& \text { with } \tilde{\mathbf{x}} \in \overline{\Omega \backslash \Omega^{(I)}\left(t_{n}\right)}, \\
\mathbf{x}=\mathbf{r}\left(t_{n-1} ; \tilde{\mathbf{x}}, \tilde{t}\right)= & \tilde{\mathbf{x}}-\mathbf{v}(\tilde{\mathbf{x}}, \tilde{t})\left(\tilde{t}-t_{n-1}\right) \quad \forall \mathbf{x} \in \Omega^{(O)}\left(t_{n-1}\right) \\
& \text { with } \quad(\tilde{\mathbf{x}}, \tilde{t}) \in \Gamma^{(O)} \times\left[t_{n-1}, t_{n}\right] .
\end{aligned}
$$

By (2.12), we bound the first term on the right-hand side of (4.2) by

$$
\begin{aligned}
& \left|\int_{\Omega} \xi\left(\mathbf{x}, t_{n-1}\right) \xi\left(\mathbf{x}, t_{n-1}^{+}\right) d \mathbf{x}\right| \\
& \quad \leq \frac{1}{2} \int_{\Omega} \xi^{2}\left(\mathbf{x}, t_{n-1}\right) d \mathbf{x}+\frac{1}{2} \int_{\Omega} \xi^{2}\left(\mathbf{x}, t_{n-1}^{+}\right) d \mathbf{x} \\
& \quad=\frac{1}{2} \int_{\Omega} \xi^{2}\left(\mathbf{x}, t_{n-1}\right) d \mathbf{x}+\frac{1}{2} \int_{\Omega \backslash \Omega^{(I)}\left(t_{n}\right)} \xi^{2}\left(\tilde{\mathbf{x}}, t_{n}\right)\left|\mathbf{J}\left(t_{n-1} ; \tilde{\mathbf{x}}, t_{n}\right)\right| d \tilde{\mathbf{x}} \\
& \quad \leq \frac{1}{2} \int_{\Omega} \xi^{2}\left(\mathbf{x}, t_{n-1}\right) d \mathbf{x}+\frac{1+M \Delta t}{2} \int_{\Omega} \xi^{2}\left(\mathbf{x}, t_{n}\right) d \mathbf{x},
\end{aligned}
$$

where at the last " $\leq$ " sign, the dummy variables $\tilde{\mathbf{x}}$ is replaced by $\mathbf{x}$.

We turn to the second and third terms on the right-hand side of (4.2). Let

$$
C r:=\max _{(\mathbf{x}, t) \in \Omega \times[0, T]}\left\{\frac{\left|V^{(1)}(\mathbf{x}, t)\right| \Delta t}{\Delta x}, \frac{\left|V^{(2)}(\mathbf{x}, t)\right| \Delta t}{\Delta y}\right\} .
$$

Case 1. $C r \geq \rho$ with $0<\rho<1$, which implies

$$
h \leq \frac{M}{\rho} \max _{(\mathbf{x}, t) \in \Omega \times[0, T]}\left\{\left|V^{(1)}(\mathbf{x}, t)\right|,\left|V^{(2)}(\mathbf{x}, t)\right|\right\} \Delta t \leq M \Delta t .
$$

Using the estimate (4.5) and the fact (4.7), we have

$$
\begin{aligned}
& \left|\int_{\Omega} \eta\left(\mathbf{x}, t_{n}\right) \xi\left(\mathbf{x}, t_{n}\right) d \mathbf{x}-\int_{\Omega} \eta\left(\mathbf{x}, t_{n-1}\right) \xi\left(\mathbf{x}, t_{n-1}^{+}\right) d \mathbf{x}\right| \\
& \quad \leq\left\|\eta\left(\mathbf{x}, t_{n}\right)\right\|_{L^{2}(\Omega)}\left\|\xi\left(\mathbf{x}, t_{n}\right)\right\|_{L^{2}(\Omega)}+\left\|\eta\left(\mathbf{x}, t_{n-1}\right)\right\|_{L^{2}(\Omega)}\left\|\xi\left(\mathbf{x}, t_{n-1}^{+}\right)\right\|_{L^{2}(\Omega)} \\
& \quad \leq M h^{2}\left\|\xi\left(\mathbf{x}, t_{n}\right)\right\|_{L^{2}(\Omega)}\|c\|_{L^{\infty}\left(0, T ; H^{2}(\Omega)\right)} \\
& \quad \leq M \Delta t\left\|\xi\left(\mathbf{x}, t_{n}\right)\right\|_{L^{2}(\Omega)}^{2}+M(\Delta t)^{3}\|c\|_{L^{\infty}\left(0, T ; H^{2}(\Omega)\right)}^{2}
\end{aligned}
$$

Case 2. $\mathrm{Cr}<\rho$ for some $0<\rho<1$. This case requires much more attention. We rewrite the second and third terms on the right-hand side of (4.2) 


$$
\begin{aligned}
& \int_{\Omega} \eta\left(\mathbf{x}, t_{n}\right) \xi\left(\mathbf{x}, t_{n}\right) d \mathbf{x}-\int_{\Omega} \eta\left(\mathbf{x}, t_{n-1}\right) \xi\left(\mathbf{x}, t_{n-1}^{+}\right) d \mathbf{x} \\
& =\int_{\Omega_{\backslash \Omega^{(O)}\left(t_{n-1}\right)}} \eta\left(\mathbf{x}, t_{n}\right) \xi\left(\mathbf{x}, t_{n}\right) d \mathbf{x}+\int_{\Omega^{(O)}\left(t_{n-1}\right)} \eta\left(\mathbf{x}, t_{n}\right) \xi\left(\mathbf{x}, t_{n}\right) d \mathbf{x} \\
& -\int_{\Omega \backslash \Omega^{(O)}\left(t_{n-1}\right)} \eta\left(\mathbf{x}, t_{n-1}\right) \xi\left(\tilde{\mathbf{x}}, t_{n}\right) d \mathbf{x}-\int_{\Omega^{(O)}\left(t_{n-1}\right)} \eta\left(\mathbf{x}, t_{n-1}\right) \xi(\tilde{\mathbf{x}}, \tilde{t}) d \mathbf{x} \\
& =\int_{\Omega \backslash \Omega^{(O)}\left(t_{n-1}\right)}\left[\eta\left(\mathbf{x}, t_{n}\right)-\eta\left(\mathbf{x}, t_{n-1}\right)\right] \xi\left(\mathbf{x}, t_{n}\right) d \mathbf{x} \\
& -\int_{\Omega \backslash \Omega^{(O)}\left(t_{n-1}\right)} \eta\left(\mathbf{x}, t_{n-1}\right)\left[\xi\left(\tilde{\mathbf{x}}, t_{n}\right)-\xi\left(\mathbf{x}, t_{n}\right)\right] d \mathbf{x} \\
& +\int_{\Omega^{(O)}\left(t_{n-1}\right)} \eta\left(\mathbf{x}, t_{n}\right) \xi\left(\mathbf{x}, t_{n}\right) d \mathbf{x} \text {. }
\end{aligned}
$$

Here we used the fact that for $\mathbf{x} \in \Omega^{(O)}\left(t_{n-1}\right),(\tilde{\mathbf{x}}, \tilde{t}) \in \Gamma^{(O)} \times\left[t_{n-1}, t_{n}\right]$. Hence, $\xi(\tilde{\mathbf{x}}, \tilde{t})=0$. So, the last term on the right-hand side of first equality vanishes.

The first term on the right-hand side of (4.9) is bounded by

$$
\begin{aligned}
& \left|\int_{\Omega \backslash \Omega^{(O)}\left(t_{n-1}\right)}\left[\eta\left(\mathbf{x}, t_{n}\right)-\eta\left(\mathbf{x}, t_{n-1}\right)\right] \xi\left(\mathbf{x}, t_{n}\right) d \mathbf{x}\right| \\
& \quad=\left|\int_{\Omega \backslash \Omega^{(O)}\left(t_{n-1}\right)}\left[\int_{t_{n-1}}^{t_{n}} \eta_{t}(\mathbf{x}, t) d t\right] \xi\left(\mathbf{x}, t_{n}\right) d \mathbf{x}\right| \\
& \quad \leq M(\Delta t)^{\frac{1}{2}}\left\|\xi\left(\mathbf{x}, t_{n}\right)\right\|_{L^{2}(\Omega)}\left\|\eta_{t}\right\|_{L^{2}\left(t_{n-1}, t_{n} ; L^{2}(\Omega)\right)} \\
& \quad \leq M \Delta t\left\|\xi\left(\mathbf{x}, t_{n}\right)\right\|_{L^{2}(\Omega)}^{2}+M h^{4}\left\|c_{t}\right\|_{L^{2}\left(t_{n-1}, t_{n} ; H^{2}(\Omega)\right)}^{2}
\end{aligned}
$$

Let $\mathbf{z}=\mathbf{x}+\theta(\tilde{\mathbf{x}}-\mathbf{x})$ and note that

$$
\left|\frac{\partial \mathbf{x}}{\partial \mathbf{z}}\right|=1+\mathcal{O}(\Delta t) .
$$

We bound the second term on the right-hand side of (4.9) as follows:

$$
\begin{aligned}
& \left|\int_{\Omega \backslash \Omega^{(O)}\left(t_{n-1}\right)} \eta\left(\mathbf{x}, t_{n-1}\right)\left[\xi\left(\tilde{\mathbf{x}}, t_{n}\right)-\xi\left(\mathbf{x}, t_{n}\right)\right] d \mathbf{x}\right| \\
& \quad=\left|\int_{\Omega \backslash \Omega^{(O)}\left(t_{n-1}\right)} \eta\left(\mathbf{x}, t_{n-1}\right)\left[\int_{0}^{1} \frac{d}{d \theta} \xi(\mathbf{x}+\theta(\tilde{\mathbf{x}}-\mathbf{x})) d \theta\right] d \mathbf{x}\right| \\
& \quad=\left|\int_{\Omega \Omega^{(O)}\left(t_{n-1}\right)} \eta\left(\mathbf{x}, t_{n-1}\right)\left[\int_{0}^{1} \nabla \xi(\mathbf{x}+\theta(\tilde{\mathbf{x}}-\mathbf{x})) d \theta\right] \cdot(\tilde{\mathbf{x}}-\mathbf{x}) d \mathbf{x}\right| \\
& \quad \leq M \Delta t\left\|\eta\left(\mathbf{x}, t_{n-1}\right)\right\|_{L^{2}(\Omega)}\left[\int_{\Omega \backslash \Omega^{(O)}\left(t_{n-1}\right)} \int_{0}^{1}|\nabla \xi(\mathbf{x}+\theta(\tilde{\mathbf{x}}-\mathbf{x}))|^{2} d \theta d \mathbf{x}\right]^{\frac{1}{2}} \\
& \quad \leq M \Delta t h^{2}\left\|c\left(\mathbf{x}, t_{n-1}\right)\right\|_{H^{2}(\Omega)}\left[\int_{\Omega}|\nabla \xi(\mathbf{z})|^{2} d \mathbf{z}\right]^{\frac{1}{2}} \\
& \quad \leq \varepsilon \Delta t\left\|\nabla \xi\left(\mathbf{x}, t_{n}\right)\right\|_{L^{2}(\Omega)}^{2}+M \Delta t h^{4}\|c\|_{L^{\infty}\left(0, T ; H^{2}(\Omega)\right)}^{2} .
\end{aligned}
$$


We need to hide the first term on the right-hand side of (4.12) by the second term on the left-hand side of (4.2). In other words, we need the following estimate:

$$
\Delta t\left\|\nabla \xi\left(\mathbf{x}, t_{n}\right)\right\|_{L^{2}(\Omega)}^{2} \leq M \int_{\Omega} \Delta t(\mathbf{x}) D\left(\mathbf{x}, t_{n}\right)\left|\nabla \xi\left(\mathbf{x}, t_{n}\right)\right|^{2} d \mathbf{x}
$$

Because a (nonphysical) uniform time step $\Delta t$ is used in the MMOC formulation, a straightforward inequality, which is similar to (4.13) but with $\Delta t(\mathbf{x})$ replaced by $\Delta t$, was used in the analysis for the MMOC [11]. Due to the degenerate time step $\Delta t(\mathbf{x})$ in the ELLAM formulation, the estimate (4.13) might not be true in general. Nevertheless, recall that we are bounding (4.12) under the condition that $C r \leq \rho<1$. In Lemma 5.1 in section 5 , we prove that there exist $0<\rho<1$ and $M_{1}=M_{1}(\rho)$, such that estimate (4.13) holds for $C r \leq \rho$. Under this assumption, we have

$$
\begin{aligned}
& \left|\int_{\Omega \backslash \Omega^{(O)}\left(t_{n-1}\right)} \eta\left(\mathbf{x}, t_{n-1}\right)\left[\xi\left(\tilde{\mathbf{x}}, t_{n}\right)-\xi\left(\mathbf{x}, t_{n}\right)\right] d \mathbf{x}\right| \\
& \quad \leq \varepsilon \int_{\Omega} \Delta t(\mathbf{x}) D\left(\mathbf{x}, t_{n}\right)\left|\nabla \xi\left(\mathbf{x}, t_{n}\right)\right|^{2} d \mathbf{x}+M \Delta t\left\|\xi\left(\mathbf{x}, t_{n}\right)\right\|_{L^{2}(\Omega)}^{2} \\
& \quad+M \Delta t h^{4}\|c\|_{L^{\infty}\left(0, T ; H^{2}(\Omega)\right)}^{2} .
\end{aligned}
$$

We are left with the last term on the right-hand side of (4.9). For $\mathbf{x} \in \Omega^{(O)}\left(t_{n-1}\right)$, there exists an $\hat{\mathbf{x}} \in \Gamma^{(O)}$ such that $\operatorname{dist}(\mathbf{x}, \hat{\mathbf{x}})=\operatorname{dist}\left(\mathbf{x}, \Gamma^{(O)}\right)=\mathcal{O}(\Delta t)$. The third equation in (2.12) implies $\xi\left(\hat{\mathbf{x}}, t_{n}\right)=0$. As in the estimate (4.12), we bound the last term on the right-hand side of (4.9) by

$$
\begin{aligned}
& \left|\int_{\Omega^{(O)}\left(t_{n-1}\right)} \eta\left(\mathbf{x}, t_{n-1}\right) \xi\left(\mathbf{x}, t_{n}\right) d \mathbf{x}\right| \\
& =\left|-\int_{\Omega^{(O)}\left(t_{n-1}\right)} \eta\left(\mathbf{x}, t_{n-1}\right)\left[\xi\left(\hat{\mathbf{x}}, t_{n}\right)-\xi\left(\mathbf{x}, t_{n}\right)\right] d \mathbf{x}\right| \\
& =\left|\int_{\Omega^{(O)}\left(t_{n-1}\right)} \eta\left(\mathbf{x}, t_{n-1}\right)\left[\int_{0}^{1} \frac{d}{d \theta} \xi(\mathbf{x}+\theta(\hat{\mathbf{x}}-\mathbf{x})) d \theta\right] d \mathbf{x}\right| \\
& \leq \varepsilon \int_{\Omega} \Delta t(\mathbf{x}) D\left(\mathbf{x}, t_{n}\right)\left|\nabla \xi\left(\mathbf{x}, t_{n}\right)\right|^{2} d \mathbf{x}+M \Delta t\left\|\xi\left(\mathbf{x}, t_{n}\right)\right\|_{L^{2}(\Omega)}^{2} \\
& \quad+M \Delta t h^{4}\|c\|_{L^{\infty}\left(0, T ; H^{2}(\Omega)\right)}^{2}
\end{aligned}
$$

Combining the estimates (4.8)-(4.10), (4.12), (4.14), and (4.15), we obtain the following estimate for the second and third terms on the right-hand side of (4.2) that holds independent of the size of $C r$ :

$$
\begin{aligned}
& \left|\int_{\Omega} \eta\left(\mathbf{x}, t_{n}\right) \xi\left(\mathbf{x}, t_{n}\right) d \mathbf{x}-\int_{\Omega} \eta\left(\mathbf{x}, t_{n-1}\right) \xi\left(\mathbf{x}, t_{n-1}^{+}\right) d \mathbf{x}\right| \\
& \quad \leq \varepsilon \int_{\Omega} \Delta t(\mathbf{x}) D\left(\mathbf{x}, t_{n}\right)\left|\nabla \xi\left(\mathbf{x}, t_{n}\right)\right|^{2} d \mathbf{x}+M \Delta t\left\|\xi\left(\mathbf{x}, t_{n}\right)\right\|_{L^{2}(\Omega)}^{2} \\
& \quad+M \Delta t\left[h^{4}+(\Delta t)^{2}\right]\|c\|_{L^{\infty}\left(0, T ; H^{2}(\Omega)\right)}^{2}+M h^{4}\left\|c_{t}\right\|_{L^{2}\left(t_{n-1}, t_{n} ; H^{2}(\Omega)\right)}^{2}
\end{aligned}
$$


The estimate for the fourth term on the right-hand side of (4.2) requires extra attention and is long. We present its proof in Lemma 6.3:

$$
\begin{aligned}
& \left|\int_{\Omega} \Delta t(\mathbf{x}) D\left(\mathbf{x}, t_{n}\right) \nabla \xi\left(\mathbf{x}, t_{n}\right) \cdot \nabla \eta\left(\mathbf{x}, t_{n}\right) d \mathbf{x}\right| \\
& \quad \leq \varepsilon \int_{\Omega} \Delta t(\mathbf{x}) D\left(\mathbf{x}, t_{n}\right)\left|\nabla \xi\left(\mathbf{x}, t_{n}\right)\right|^{2} d \mathbf{x}+M \Delta t\left\|\xi\left(\mathbf{x}, t_{n}\right)\right\|_{L^{2}(\Omega)}^{2} \\
& \quad+M \Delta t\left[h^{4}+(\Delta t)^{2}\right]\|c\|_{L^{\infty}\left(0, T ; W^{2, \infty}(\Omega)\right)}^{2}
\end{aligned}
$$

Finally, we turn to the last term on the right-hand side of (4.2). Using (2.16), (2.18), and (2.20), we write this term as

$$
\begin{aligned}
& E(D, f, c, \xi) \\
& =-\int_{\Omega}\left[\int_{t^{*}(\mathbf{x})}^{t_{n}}\left[f\left(\mathbf{x}, t_{n}\right)-f\left(\mathbf{r}\left(\theta ; \mathbf{x}, t_{n}\right), \theta\right)\left|\mathbf{J}\left(\theta ; \mathbf{x}, t_{n}\right)\right|\right] d \theta\right] \xi\left(\mathbf{x}, t_{n}\right) d \mathbf{x} \\
& \quad-\int_{\Omega} \int_{t^{*}(\mathbf{x})}^{t_{n}}\left[\nabla \xi\left(\mathbf{x}, t_{n}\right) \cdot(D \nabla c)\left(\mathbf{x}, t_{n}\right)\right. \\
& \left.\quad-\nabla \xi\left(\mathbf{r}\left(\theta ; \mathbf{x}, t_{n}\right), \theta\right) \cdot(D \nabla c)\left(\mathbf{r}\left(\theta ; \mathbf{x}, t_{n}\right), \theta\right)\left|\mathbf{J}\left(\theta ; \mathbf{x}, t_{n}\right)\right|\right] d \theta d \mathbf{x} \\
& \quad+\int_{t_{n-1}}^{t_{n}} \int_{\Omega} c(\mathbf{z}, \theta)\left(\xi_{\theta}+\mathbf{v} \cdot \nabla \xi\right)(\mathbf{z}, \theta) d \mathbf{z} d \theta
\end{aligned}
$$

Using (2.15) and (2.16), we bound the first term by

$$
\begin{aligned}
& \left|\int_{\Omega}\left[\int_{t^{*}(\mathbf{x})}^{t_{n}}\left[f\left(\mathbf{x}, t_{n}\right)-f\left(\mathbf{r}\left(\theta ; \mathbf{x}, t_{n}\right), \theta\right)\left|\mathbf{J}\left(\theta ; \mathbf{x}, t_{n}\right)\right|\right] d \theta\right] \xi\left(\mathbf{x}, t_{n}\right) d \mathbf{x}\right| \\
& \leq\left|\int_{\Omega}\left\{\int_{t^{*}(\mathbf{x})}^{t_{n}}\left[f\left(\mathbf{x}, t_{n}\right)-f\left(\mathbf{r}\left(\theta ; \mathbf{x}, t_{n}\right), \theta\right)\right] d \theta\right\} \xi\left(\mathbf{x}, t_{n}\right) d \mathbf{x}\right| \\
& +\left|\int_{\Omega}\left\{\int_{t^{*}(\mathbf{x})}^{t_{n}} \mathcal{O}\left(t_{n}-\theta\right) f\left(\mathbf{r}\left(\theta ; \mathbf{x}, t_{n}\right), \theta\right) d \theta\right\} \xi\left(\mathbf{x}, t_{n}\right) d \mathbf{x}\right| \\
& \leq M(\Delta t)^{2}\left[\left\|\frac{d f}{d \theta}\right\|_{L^{2}\left(t_{n-1}, t_{n} ; L^{2}(\Omega)\right)}^{2}+\|f\|_{L^{2}\left(t_{n-1}, t_{n} ; L^{2}(\Omega)\right)}^{2}\right] \\
& \quad+M \Delta t\left\|\xi\left(\mathbf{x}, t_{n}\right)\right\|_{L^{2}(\Omega)}^{2} .
\end{aligned}
$$

Utilizing the relationship

$$
\begin{array}{rlrl}
\nabla \xi\left(\mathbf{r}\left(\theta ; \mathbf{x}, t_{n}\right), \theta\right) & :=\nabla_{\mathbf{r}} \xi\left(\mathbf{r}\left(\theta ; \mathbf{x}, t_{n}\right), \theta\right) & & =\nabla_{\mathbf{r}} \xi\left(\mathbf{x}, t_{n}\right) \\
& =\frac{\partial \mathbf{x}}{\partial \mathbf{r}\left(\theta ; \mathbf{x}, t_{n}\right)} \nabla_{\mathbf{x}} \xi\left(\mathbf{x}, t_{n}\right) & & =\mathbf{J}^{-1}\left(\theta ; \mathbf{x}, t_{n}\right) \nabla_{\mathbf{x}} \xi\left(\mathbf{x}, t_{n}\right) \\
& =\left(1+\left(t_{n}-\theta\right)\right) \nabla_{\mathbf{x}} \xi\left(\mathbf{x}, t_{n}\right) & \forall \mathbf{x} \in \bar{\Omega}
\end{array}
$$


we bound the second term on the right-hand side of (4.18) by

$$
\begin{aligned}
& \mid \int_{\Omega} \int_{t^{*}(\mathbf{x})}^{t_{n}}\left[\nabla \xi\left(\mathbf{x}, t_{n}\right) \cdot(D \nabla c)\left(\mathbf{x}, t_{n}\right)\right. \\
& \left.\quad-\nabla \xi\left(\mathbf{r}\left(\theta ; \mathbf{x}, t_{n}\right), \theta\right) \cdot(D \nabla c)\left(\mathbf{r}\left(\theta ; \mathbf{x}, t_{n}\right), \theta\right)\left|\mathbf{J}\left(\theta ; \mathbf{x}, t_{n}\right)\right|\right] d \theta d \mathbf{x} \mid \\
& =\mid \int_{\Omega} \nabla \xi\left(\mathbf{x}, t_{n}\right) \cdot\left[\int_{t^{*}(\mathbf{x})}^{t_{n}} \int_{\theta}^{t_{n}} \frac{d}{d \gamma}(D \nabla c)\left(\mathbf{r}\left(\gamma ; \mathbf{x}, t_{n}\right), \gamma\right) d \gamma d \theta\right] d \mathbf{x} \\
& \quad+\int_{\Omega} \nabla \xi\left(\mathbf{x}, t_{n}\right) \cdot\left[\int_{t^{*}(\mathbf{x})}^{t_{n}}(D \nabla c)\left(\mathbf{r}\left(\theta ; \mathbf{x}, t_{n}\right), \theta\right) \mathcal{O}(\Delta t(\mathbf{x})) d \theta\right] d \mathbf{x} \mid \\
& \leq \varepsilon \int_{\Omega} \Delta t(\mathbf{x}) D\left(\mathbf{x}, t_{n}\right)\left|\nabla \xi\left(\mathbf{x}, t_{n}\right)\right|^{2} d \mathbf{x} \\
& \quad+M(\Delta t)^{2}\left[\left\|\frac{d c}{d \theta}\right\|_{L^{2}\left(t_{n-1}, t_{n} ; H^{1}(\Omega)\right)}^{2}+\|c\|_{L^{2}\left(t_{n-1}, t_{n} ; H^{1}(\Omega)\right)}^{2}\right]
\end{aligned}
$$

At first glance, the only potential convergence factor in the last term on the righthand side of (4.18) is the outside integral from $t_{n-1}$ to $t_{n}$. This factor will disappear as one sums the estimate for all the time levels later. Nevertheless, because the test functions $w$ are constant along the approximate characteristics (2.9), they satisfy a first-order approximation to (2.8). Using (3.10), we have

$$
\begin{array}{r}
\xi_{\theta}\left(\mathbf{r}\left(\theta ; \mathbf{x}, t_{n}\right), \theta\right)+\mathbf{v}\left(\mathbf{x}, t_{n}\right) \cdot \nabla \xi\left(\mathbf{r}\left(\theta ; \mathbf{x}, t_{n}\right), \theta\right) \quad=0 \\
\theta \in\left[t^{*}(\mathbf{x}), t_{n}\right], \quad \mathbf{x} \in \bar{\Omega} .
\end{array}
$$

Using (4.22), we bound the first term in a similar manner to (2.14) by

$$
\begin{aligned}
& \left|\int_{t_{n-1}}^{t_{n}} \int_{\Omega} c(\mathbf{z}, \theta)\left(\xi_{\theta}+\mathbf{v} \cdot \nabla \xi\right)(\mathbf{z}, \theta) d \mathbf{z} d \theta\right| \\
& =\left|\int_{\Omega} \int_{t^{*}(\mathbf{x})}^{t_{n}} c\left(\mathbf{r}\left(\theta ; \mathbf{x}, t_{n}\right), \theta\right)\left(\xi_{\theta}+\mathbf{v} \cdot \nabla \xi\right)\left(\mathbf{r}\left(\theta ; \mathbf{x}, t_{n}\right), \theta\right)\right| \mathbf{J}\left(\theta ; \mathbf{x}, t_{n}\right) \mid d \theta d \mathbf{x} \\
& \quad-\int_{\Omega} \int_{t^{*}(\mathbf{x})}^{t_{n}} c\left(\mathbf{r}\left(\theta ; \mathbf{x}, t_{n}\right), \theta\right)\left(\xi_{\theta}\left(\mathbf{r}\left(\theta ; \mathbf{x}, t_{n}\right), \theta\right)\right. \\
& \left.\quad+\mathbf{v}\left(\mathbf{x}, t_{n}\right) \cdot \nabla \xi\left(\mathbf{r}\left(\theta ; \mathbf{x}, t_{n}\right), \theta\right)\right)\left|\mathbf{J}\left(\theta ; \mathbf{x}, t_{n}\right)\right| d \theta d \mathbf{x} \mid \\
& =\mid \int_{\Omega} \int_{t^{*}(\mathbf{x})}^{t_{n}}\left(\mathbf{v}\left(\mathbf{x}, t_{n}\right)-\mathbf{v}\left(\mathbf{r}\left(\theta ; \mathbf{x}, t_{n}\right), \theta\right)\right) \cdot(c \nabla \xi)\left(\mathbf{r}\left(\theta ; \mathbf{x}, t_{n}\right), \theta\right) \\
& \quad \times\left|\mathbf{J}\left(\theta ; \mathbf{x}, t_{n}\right)\right| d \theta d \mathbf{x} \mid
\end{aligned}
$$




$$
\begin{aligned}
& =\mid \int_{\Omega} \int_{t^{*}(\mathbf{x})}^{t_{n}}\left[\int_{\theta}^{t_{n}} \frac{d}{d \gamma} \mathbf{v}\left(\mathbf{r}\left(\gamma ; \mathbf{x}, t_{n}\right), \gamma\right) d \gamma\right] \cdot(c \nabla \xi)\left(\mathbf{r}\left(\theta ; \mathbf{x}, t_{n}\right), \theta\right) \\
& \quad \times\left|\mathbf{J}\left(\theta ; \mathbf{x}, t_{n}\right)\right| d \theta d \mathbf{x} \mid \\
& \leq \varepsilon \int_{\Omega} \Delta t(\mathbf{x}) D\left(\mathbf{x}, t_{n}\right)\left|\nabla \xi\left(\mathbf{x}, t_{n}\right)\right|^{2} d \mathbf{x}+M(\Delta t)^{2}\|c\|_{L^{2}\left(t_{n-1}, t_{n} ; L^{2}(\Omega)\right)}^{2}
\end{aligned}
$$

Incorporating the estimates (4.19), (4.21), and (4.23) into (4.18), we obtain the following estimate for the last term on the right-hand side of (5.2):

$$
\begin{aligned}
E(D, f, c, \xi) \leq & \varepsilon \int_{\Omega} \Delta t(\mathbf{x}) D\left(\mathbf{x}, t_{n}\right)\left|\nabla \xi\left(\mathbf{x}, t_{n}\right)\right|^{2} d \mathbf{x}+M \Delta t\left\|\xi\left(\mathbf{x}, t_{n}\right)\right\|_{L^{2}(\Omega)}^{2} \\
& +M(\Delta t)^{2}\left[\left\|\frac{d c}{d \theta}\right\|_{L^{2}\left(t_{n-1}, t_{n} ; H^{1}(\Omega)\right)}^{2}+\|c\|_{L^{2}\left(t_{n-1}, t_{n} ; H^{1}(\Omega)\right)}^{2}+\|f\|_{L^{2}\left(t_{n-1}, t_{n} ; L^{2}(\Omega)\right)}^{2}\right] . \\
& +\left\|\frac{d f}{d \theta}\right\|_{L^{2}\left(t_{n-1}, t_{n} ; L^{2}(\Omega)\right)}^{2}+.
\end{aligned}
$$

Substituting the estimates (4.5), (4.16), (4.17), and (4.24) for the corresponding terms in (4.2), we come up with the following estimates:

$$
\begin{aligned}
& \int_{\Omega} \xi^{2}\left(\mathbf{x}, t_{n}\right) d \mathbf{x}+\int_{\Omega} \Delta t(\mathbf{x}) D\left(\mathbf{x}, t_{n}\right)\left|\nabla \xi\left(\mathbf{x}, t_{n}\right)\right|^{2} d \mathbf{x} \\
& \leq \frac{1+M \Delta t}{2} \int_{\Omega} \xi^{2}\left(\mathbf{x}, t_{n}\right) d \mathbf{x}+\varepsilon \int_{\Omega} \Delta t(\mathbf{x}) D\left(\mathbf{x}, t_{n}\right)\left|\nabla \xi\left(\mathbf{x}, t_{n}\right)\right|^{2} d \mathbf{x} \\
& \quad+\frac{1}{2} \int_{\Omega} \xi^{2}\left(\mathbf{x}, t_{n-1}\right) d \mathbf{x}+M \Delta t\left[h^{4}+(\Delta t)^{2}\right]\|c\|_{L^{\infty}\left(0, T ; W^{2, \infty}(\Omega)\right)}^{2} \\
& \quad+M h^{4}\left\|c_{t}\right\|_{L^{2}\left(t_{n-1}, t_{n} ; H^{2}(\Omega)\right)}^{2}+M(\Delta t)^{2}\left[\left\|\frac{d c}{d \theta}\right\|_{L^{2}\left(t_{n-1}, t_{n} ; H^{1}(\Omega)\right)}^{2}+\|f\|_{L^{2}\left(t_{n-1}, t_{n} ; L^{2}(\Omega)\right)}^{2}\right] .
\end{aligned}
$$

Taking $\varepsilon=\frac{1}{2}$, summing the inequality (4.25) from $n=1$ to $n=N_{1}\left(N_{1} \leq N\right)$, canceling the corresponding terms, and multiplying both sides by $\frac{1}{2}$, we obtain

$$
\begin{aligned}
\int_{\Omega} \xi^{2}\left(\mathbf{x}, t_{N_{1}}\right) d \mathbf{x}+\sum_{n=0}^{N_{1}} \int_{\Omega} \Delta t(\mathbf{x}) D\left(\mathbf{x}, t_{n}\right)\left|\nabla \xi\left(\mathbf{x}, t_{n}\right)\right|^{2} d \mathbf{x} \\
\leq M \Delta t \sum_{n=0}^{N_{1}}\left\|\xi\left(\mathbf{x}, t_{n}\right)\right\|_{L^{2}(\Omega)}^{2}+M(\Delta t)^{2}\left[\left\|\frac{d c}{d \theta}\right\|_{L^{2}\left(0, T ; H^{1}(\Omega)\right)}^{2}+\|f\|_{L^{2}\left(0, T ; L^{2}(\Omega)\right)}^{2}\right] \\
\quad+\|c\|_{L^{2}\left(0, T ; H^{1}(\Omega)\right)}^{2}+\left\|\frac{d f}{d \theta}\right\|_{L^{2}\left(0, T ; L^{2}(\Omega)\right)}^{2}+\left\|h^{4}\right\| c_{t} \|_{L^{2}\left(0, T ; H^{2}(\Omega)\right)}^{2} \\
\quad+M\left[h^{4}+(\Delta t)^{2}\right]\|c\|_{L^{\infty}\left(0, T ; W^{2, \infty}(\Omega)\right)}^{2}+M h^{4}
\end{aligned}
$$


Choosing $\Delta t$ to satisfy $M \Delta t \leq \frac{1}{2}$ and using Gronwall's inequality, we have

$$
\begin{aligned}
& \|\xi\|_{\hat{L}^{\infty}\left(0, T ; L^{2}(\Omega)\right)}+\left[\sum_{n=0}^{N} \int_{\Omega} \Delta t(\mathbf{x}) D\left(\mathbf{x}, t_{n}\right)\left|\nabla \xi\left(\mathbf{x}, t_{n}\right)\right|^{2} d \mathbf{x}\right]^{\frac{1}{2}} \\
& \leq M \Delta t\left[\left\|\frac{d c}{d \theta}\right\|_{L^{2}\left(0, T ; H^{1}(\Omega)\right)}+\|c\|_{L^{\infty}\left(0, T ; W^{2, \infty}(\Omega)\right)}+\left\|\frac{d f}{d \theta}\right\|_{L^{2}\left(0, T ; L^{2}(\Omega)\right)}\right. \\
& \left.\quad+\|f\|_{L^{2}\left(0, T ; L^{2}(\Omega)\right)}\right]+M h^{2}\left[\|c\|_{L^{\infty}\left(0, T ; W^{2, \infty}(\Omega)\right)}+\left\|c_{t}\right\|_{L^{2}\left(0, T ; H^{2}(\Omega)\right)}\right] .
\end{aligned}
$$

The combination of (4.27) and (3.7) yields the main result of this paper.

THEOREM 4.1. Under Assumption (A), the following optimal-order $L^{2}$ error estimate holds:

$$
\begin{aligned}
& \|C-c\|_{\hat{L}^{\infty}\left(0, T ; L^{2}(\Omega)\right)} \\
& \leq M \Delta t\left[\left\|\frac{d c}{d \theta}\right\|_{L^{2}\left(0, T ; H^{1}(\Omega)\right)}+\|c\|_{L^{\infty}\left(0, T ; W^{2, \infty}(\Omega)\right)}+\left\|\frac{d f}{d \theta}\right\|_{L^{2}\left(0, T ; L^{2}(\Omega)\right)}\right. \\
& \left.\quad+\|f\|_{L^{2}\left(0, T ; L^{2}(\Omega)\right)}\right]+M h^{2}\left[\|c\|_{L^{\infty}\left(0, T ; W^{2, \infty}(\Omega)\right)}+\left\|c_{t}\right\|_{L^{2}\left(0, T ; H^{2}(\Omega)\right)}\right] .
\end{aligned}
$$

Furthermore, the superconvergence estimate holds if $c \in L^{\infty}\left(0, T ; H^{3}(\Omega)\right)$

$$
\begin{aligned}
\| C- & c \|_{\hat{L}^{\infty}\left(0, T ; \hat{H}^{1}(\Omega)\right)} \\
\leq & M \Delta t\left[\left\|\frac{d c}{d \theta}\right\|_{L^{2}\left(0, T ; H^{1}(\Omega)\right)}+\|c\|_{L^{\infty}\left(0, T ; W^{2, \infty}(\Omega)\right)}+\left\|\frac{d f}{d \theta}\right\|_{L^{2}\left(0, T ; L^{2}(\Omega)\right)}\right. \\
& \left.+\|f\|_{L^{2}\left(0, T ; L^{2}(\Omega)\right)}\right]+M h^{2}\left[\|c\|_{L^{\infty}\left(0, T ; W^{2, \infty}(\Omega)\right)}+\left\|c_{t}\right\|_{L^{2}\left(0, T ; H^{2}(\Omega)\right)}\right. \\
& \left.+\|c\|_{L^{\infty}\left(0, T ; H^{3}(\Omega)\right)}\right] .
\end{aligned}
$$

5. Proof of the estimate (4.13). In this section, we prove the estimate (4.13) which is used repeatedly in the analyses of this paper.

LEMMA 5.1. Suppose the coefficient $D(\mathbf{x}, t)$ in (2.1) satisfies Assumption (A). Then there exist positive constants $0<\rho<1$ and $M_{1}=M_{1}(\rho)$ such that

$$
\Delta t\left\|\nabla \xi\left(\mathbf{x}, t_{n}\right)\right\|_{L^{2}(\Omega)}^{2} \leq M_{1} \int_{\Omega} \Delta t(\mathbf{x}) D\left(\mathbf{x}, t_{n}\right)\left|\nabla \xi\left(\mathbf{x}, t_{n}\right)\right|^{2} d \mathbf{x}
$$

holds for $C r<\rho$ with $C r$ being defined in (4.6).

Proof. Without loss of generality, we assume that the left and the bottom sides of the domain $\Omega$ are the inflow boundary while the right and the top sides are the outflow boundary

$$
\begin{aligned}
& \Gamma^{(I)}=\left\{(x, y) \mid x=x^{L} \forall y \in\left[y^{L}, y^{R}\right] \text { or } y=y^{L} \forall x \in\left[x^{L}, x^{R}\right]\right\}, \\
& \Gamma^{(O)}=\left\{(x, y) \mid x=x^{R} \forall y \in\left[y^{L}, y^{R}\right] \text { or } y=y^{R} \forall x \in\left[x^{L}, x^{R}\right]\right\} .
\end{aligned}
$$


Using the fact that $\Delta t(\mathbf{x})=\Delta t$ on $\left(x_{0}+C r \Delta t, x_{I}\right) \times\left(y_{0}+C r \Delta t, y_{J}\right)$, for $C r \leq$ $\rho<1$ we rewrite the second term on the left-hand side of (4.2) as follows:

$$
\begin{aligned}
\int_{\Omega} \Delta t(\mathbf{x}) D\left(\mathbf{x}, t_{n}\right)\left|\nabla \xi\left(\mathbf{x}, t_{n}\right)\right|^{2} d \mathbf{x} \geq & D_{\min } \Delta t\left\|\nabla \xi\left(\mathbf{x}, t_{n}\right)\right\|_{L^{2}\left(\left(x_{1}, x_{I}\right) \times\left(y_{1}, y_{J}\right)\right)}^{2} \\
& +D_{\min } \int_{y_{1}}^{y_{J}} \int_{x_{0}}^{x_{1}} \Delta t(\mathbf{x})\left|\nabla \xi\left(\mathbf{x}, t_{n}\right)\right|^{2} d \mathbf{x} \\
& +D_{\min } \int_{y_{0}}^{y_{1}} \int_{x_{0}}^{x_{1}} \Delta t(\mathbf{x})\left|\nabla \xi\left(\mathbf{x}, t_{n}\right)\right|^{2} d \mathbf{x} \\
& +D_{\min } \int_{y_{0}}^{y_{1}} \int_{x_{1}}^{x_{I}} \Delta t(\mathbf{x})\left|\nabla \xi\left(\mathbf{x}, t_{n}\right)\right|^{2} d \mathbf{x} .
\end{aligned}
$$

Because $\xi_{x}\left(x, y, t_{n}\right)$ is constant in $x$ on the interval $\left[x_{0}, x_{1}\right]$, we bound the second term on the right-hand side of (5.3) from below by

$$
\begin{aligned}
\int_{y_{1}}^{y_{J}} \int_{x_{0}}^{x_{1}} \Delta t(\mathbf{x}) \xi_{x}^{2}\left(\mathbf{x}, t_{n}\right) d \mathbf{x} & =\int_{y_{1}}^{y_{J}}\left[\int_{x_{0}}^{x_{1}} \Delta t(x, y) d x\right] \xi_{x}^{2}\left(x, y, t_{n}\right) d y \\
& \geq \int_{y_{1}}^{y_{J}}\left[\int_{x_{0}+C r \Delta x}^{x_{1}} \Delta t d x\right] \xi_{x}^{2}\left(x, y, t_{n}\right) d y \\
& =(1-C r) \Delta t \int_{y_{1}}^{y_{J}} \int_{x_{0}}^{x_{1}} \xi_{x}^{2}\left(x, y, t_{n}\right) d x d y
\end{aligned}
$$

Note that $\xi_{y}\left(x, y, t_{n}\right)$ is linear in $x$ on the interval $\left[x_{0}, x_{1}\right]$ and that any linear function $q(x)$ has the following equivalent norms:

$$
\frac{\Delta x}{6}\left(q^{2}\left(x_{0}\right)+q^{2}\left(x_{1}\right)\right) \leq \int_{x_{0}}^{x_{1}} q^{2}(x) d x \leq \frac{\Delta x}{2}\left(q^{2}\left(x_{0}\right)+q^{2}\left(x_{1}\right)\right),
$$

we have the estimate

$$
\begin{aligned}
\int_{y_{1}}^{y_{J}} & \int_{x_{0}}^{x_{1}} \Delta t(\mathbf{x}) \xi_{y}^{2}\left(\mathbf{x}, t_{n}\right) d \mathbf{x} \\
= & \Delta t \int_{y_{1}}^{y_{J}} \int_{x_{0}}^{x_{1}} \xi_{y}^{2}\left(x, y, t_{n}\right) d x d y \\
& -\int_{y_{1}}^{y_{J}} \int_{x_{0}}^{x_{0}+C r \Delta x}\left(t^{*}(x, y)-t_{n-1}\right) \xi_{y}^{2}\left(x, y, t_{n}\right) d x d y \\
\geq & \frac{1}{6} \Delta t \Delta x \int_{y_{1}}^{y_{J}}\left(\xi_{y}^{2}\left(x_{0}, y, t_{n}\right)+\xi_{y}^{2}\left(x_{1}, y, t_{n}\right)\right) d y \\
& -C r \Delta t \Delta x \int_{y_{1}}^{y_{J}} \max _{x \in\left[x_{0}, x_{1}\right]} \xi_{y}^{2}\left(x, y, t_{n}\right) d y \\
\geq & \left(\frac{1}{6}-C r\right) \Delta t \Delta x \int_{y_{1}}^{y_{J}}\left(\xi_{y}^{2}\left(x_{0}, y, t_{n}\right)+\xi_{y}^{2}\left(x_{1}, y, t_{n}\right)\right) d y \\
\geq & \left(\frac{1}{3}-2 C r\right) \Delta t \int_{y_{1}}^{y_{J}} \int_{x_{0}}^{x_{1}} \xi_{y}^{2}\left(x, y, t_{n}\right) d x d y .
\end{aligned}
$$

Combining the estimates (5.4) and (5.7), we obtain a lower bound for the second term on the right-hand side of $(5.3)$ for $0<C r \leq \rho<\frac{1}{6}$

$$
\int_{y_{1}}^{y_{J}} \int_{x_{0}}^{x_{1}} \Delta t(\mathbf{x})\left|\nabla \xi\left(\mathbf{x}, t_{n}\right)\right|^{2} d \mathbf{x} \geq\left(\frac{1}{3}-2 \rho\right) \Delta t\left\|\nabla \xi\left(\mathbf{x}, t_{n}\right)\right\|_{L^{2}\left(\left(x_{0}, x_{1}\right) \times\left(y_{1}, y_{J}\right)\right)}^{2}
$$


By symmetry, the same estimate holds for the last term on the right-hand side of (5.3) for $0<C r \leq \rho<\frac{1}{6}$

$\int_{x_{1}}^{x_{I}} \int_{y_{0}}^{y_{1}} \Delta t(\mathbf{x})\left|\nabla \xi\left(\mathbf{x}, t_{n}\right)\right|^{2} d \mathbf{x} \geq\left(\frac{1}{3}-2 \rho\right) \Delta t\left\|\nabla \xi\left(\mathbf{x}, t_{n}\right)\right\|_{L^{2}\left(\left(x_{1}, x_{I}\right) \times\left(y_{0}, y_{1}\right)\right)}^{2}$

The $\xi_{x}$ part in the third term on the right-hand side of (5.3) is rewritten

$$
\begin{aligned}
\int_{y_{0}}^{y_{1}} & \int_{x_{0}}^{x_{1}} \Delta t(\mathbf{x}) \xi_{x}^{2}\left(\mathbf{x}, t_{n}\right) d \mathbf{x} \\
= & \int_{x_{0}+C r \Delta x}^{x_{1}} \int_{y_{0}}^{y_{1}} \Delta t(\mathbf{x}) \xi_{x}^{2}\left(\mathbf{x}, t_{n}\right) d \mathbf{x} \\
& +\int_{y_{0}}^{y_{1}}\left[\int_{x_{0}}^{x_{0}+C r \Delta x} \Delta t(x, y) d x\right] \xi_{x}^{2}\left(x, y, t_{n}\right) d y .
\end{aligned}
$$

The first term on the right-hand side is bounded in a similar manner to the estimate (5.6) but with the role of $x$ and $y$ reversed, yielding

$$
\begin{aligned}
& \int_{x_{0}+C r \Delta x}^{x_{1}} \int_{y_{0}}^{y_{1}} \Delta t(\mathbf{x}) \xi_{x}^{2}\left(\mathbf{x}, t_{n}\right) d \mathbf{x} \\
& \quad \geq\left(\frac{1}{3}-2 \rho\right) \Delta t\left\|\xi_{x}\left(\mathbf{x}, t_{n}\right)\right\|_{L^{2}\left(\left(x_{0}+C r \Delta x, x_{1}\right) \times\left(y_{0}, y_{1}\right)\right)}^{2}
\end{aligned}
$$

For $(x, y) \in\left[x_{0}, x_{0}+C r \Delta x\right] \times\left[y_{0}+C r \Delta y, y_{1}\right]$, the characteristic $\mathbf{r}\left(\theta ; x, y, t_{n}\right)=$ $\left(r^{(1)}\left(\theta ; x, y, t_{n}\right), r^{(2)}\left(\theta ; x, y, t_{n}\right)\right)$ defined by $(2.9)$ will either stay in $\Omega$ at time $t_{n-1}$ or backtrack to the boundary $x=x_{0}$ during the time period $\left[t_{n-1}, t_{n}\right]$. For $(x, y) \in$ $\left[x_{0}, x_{0}+C r \Delta x\right] \times\left[y_{0}, y_{0}+C r \Delta y\right], \mathbf{r}\left(\theta ; x, y, t_{n}\right)$ may stay inside $\Omega$ at time $t_{n-1}$ or backtrack to the boundary $x=x_{0}$ or $y=y_{0}$ during the time period $\left[t_{n-1}, t_{n}\right]$. For $y \in\left[y_{0}+C r \Delta t, y_{J}\right]$, let $\tilde{x}(y)$ be defined by

$$
\begin{gathered}
x=r^{(1)}\left(t_{n-1} ; \tilde{x}(y), y, t_{n}\right)=\tilde{x}(y)-V^{(1)}\left(\tilde{x}(y), y, t_{n}\right) \Delta t \\
\forall y \in\left[y_{0}+C r \Delta t, y_{J}\right] .
\end{gathered}
$$

We use (6.4) in section 6 to rewrite the second term on the right-hand side of (5.9)

$$
\begin{aligned}
\int_{y_{0}}^{y_{1}} & {\left[\int_{x_{0}}^{x_{0}+C r \Delta x} \Delta t(x, y) d x\right] \xi_{x}^{2}\left(x, y, t_{n}\right) d y } \\
= & \int_{y_{0}}^{y_{0}+C r \Delta y}\left[\int_{x_{0}}^{x_{0}+C r \Delta x}\left(\Delta t-\left(t^{*}(x, y)-t_{n-1}\right)\right) d x\right] \xi_{x}^{2}\left(x, y, t_{n}\right) d y \\
& +\int_{y_{0}+C r \Delta y}^{y_{1}}\left[\int_{x_{0}}^{\tilde{x}_{0}(y)} \frac{[1+\mathcal{O}(\Delta t)]\left(x-x_{0}\right)}{V^{(1)}\left(\tilde{x}_{0}(y), y, t_{n}\right)} d x\right. \\
& \left.+\int_{\tilde{x}_{0}(y)}^{x_{0}+C r \Delta x} \Delta t d x\right] \xi_{x}^{2}\left(x, y, t_{n}\right) d y .
\end{aligned}
$$




$$
\begin{gathered}
\int_{x_{0}}^{\tilde{x}_{0}(y)} \frac{[1+\mathcal{O}(\Delta t)]\left(x-x_{0}\right)}{V^{(1)}\left(\tilde{x}_{0}(y), y, t_{n}\right)} d x+\int_{\tilde{x}_{0}(y)}^{x_{0}+C r \Delta x} \Delta t d x \\
\quad \geq \frac{(1-M \Delta t)\left(\tilde{x}_{0}(y)-x_{0}\right)^{2}}{2 V^{(1)}\left(\tilde{x}_{0}(y), y, t_{n}\right)}+\int_{\tilde{x}_{0}(y)}^{x_{0}+C r \Delta x} \Delta t d x \\
=\frac{(1-M \Delta t) \Delta t}{2} \int_{x_{0}}^{\tilde{x}_{0}(y)} d x+\int_{\tilde{x}_{0}(y)}^{x_{0}+C r \Delta x} \Delta t d x \\
\geq \frac{(1-M \Delta t) \Delta t}{2} \int_{x_{0}}^{x_{0}+C r \Delta x} \Delta t d x .
\end{gathered}
$$

We use the inverse inequality $(3.8)$ on $\left[x_{0}, x_{1}\right] \times\left[y_{0}, y_{1}\right]$ to bound a part of the first term on the right-hand side of (5.12) by

$$
\begin{aligned}
& \left|\int_{y_{0}}^{y_{0}+C r \Delta y}\left[\int_{x_{0}}^{x_{0}+C r \Delta x}\left(t^{*}(x, y)-t_{n-1}\right) d x\right] \xi_{x}^{2}\left(x, y, t_{n}\right) d y\right| \\
& \quad \leq \rho^{2} \Delta t \Delta x \Delta y\left\|\xi_{x}\left(\mathbf{x}, t_{n}\right)\right\|_{L^{\infty}\left(\left(x_{0}, x_{0}+C r \Delta x\right) \times\left(y_{0}, y_{1}\right)\right)}^{2} \\
& \quad \leq M_{2} \rho^{2} \Delta t\left\|\xi_{x}\left(\mathbf{x}, t_{n}\right)\right\|_{L^{2}\left(\left(x_{0}, x_{1}\right) \times\left(y_{0}, y_{1}\right)\right)}^{2}
\end{aligned}
$$

Combining the estimates (5.9) through (5.14), we have

$$
\begin{aligned}
& \int_{y_{0}}^{y_{1}} \int_{x_{0}}^{x_{1}} \Delta t(\mathbf{x}) \xi_{x}^{2}\left(\mathbf{x}, t_{n}\right) d \mathbf{x} \\
& \geq\left(\min \left\{\frac{1}{3}-2 \rho, \frac{1-M \Delta t}{2}\right\}-M_{2} \rho^{2}\right) \Delta t\left\|\xi_{x}\left(\mathbf{x}, t_{n}\right)\right\|_{L^{2}\left(\left(x_{0}, x_{1}\right) \times\left(y_{0}, y_{1}\right)\right)}^{2} \\
& \geq \frac{1}{6} \Delta t\left\|\xi_{x}\left(\mathbf{x}, t_{n}\right)\right\|_{L^{2}\left(\left(x_{0}, x_{1}\right) \times\left(y_{0}, y_{1}\right)\right)}^{2}
\end{aligned}
$$

for $\rho$ satisfying $2 \rho+M_{2} \rho^{2}<\frac{1}{6}$. By symmetry, under the same constraint we have

$$
\int_{y_{0}}^{y_{1}} \int_{x_{0}}^{x_{1}} \Delta t(\mathbf{x}) \xi_{y}^{2}\left(\mathbf{x}, t_{n}\right) d \mathbf{x} \geq \frac{1}{6} \Delta t\left\|\xi_{y}\left(\mathbf{x}, t_{n}\right)\right\|_{L^{2}\left(\left(x_{0}, x_{1}\right) \times\left(y_{0}, y_{1}\right)\right)}^{2} .
$$

Incorporating the estimates (5.7), (5.8), (5.15), and (5.16) into (5.3), we prove the estimate (5.1).

6. Proof of the estimate (4.17). In this section, we derive the estimate (4.17) in Lemma 6.3 which gives an upper bound for the fourth term on the right-hand side of (4.2). This proof is based on Lemmas 6.1 and 6.2.

LEMma 6.1. Let $V(x, t), \tilde{x}, t^{*}(x), \Delta t(x)$, and $C x$ be one-dimensional analogues of $\mathbf{v}(\mathbf{x}, t), \tilde{\mathbf{x}}, t^{*}(\mathbf{x}), \Delta t(\mathbf{x})$, and $C r$ defined by

$$
\begin{array}{lll}
x & =r\left(t_{n-1} ; \tilde{x}, t_{n}\right) \quad:=\tilde{x}-V\left(\tilde{x}, t_{n-1}\right) \Delta t, \\
x_{0} & =r\left(t^{*}(x) ; x, t_{n}\right) \quad:=x-V\left(x, t^{*}(x)\right) \Delta t(x) \quad \forall x \in\left[x_{0}, \tilde{x}_{0}\right], \\
\Delta t(x) & :=t_{n}-t^{*}(x), \\
C x & :=\frac{\tilde{x}_{0}-x_{0}}{\Delta x},
\end{array}
$$

with $t^{*}(x)=t_{n-1}$ for any $x \in\left[\tilde{x}_{0}, x_{I}\right]$. Let $I c=\lceil C x\rceil$ be the smallest integer that is larger than or equal to $C x$, and $\psi(x)$ be any piecewise-linear function defined on 
$\left[x_{0}, x_{I}\right]$ with the spatial partition in (2.4). Then, we have the estimates

$$
\begin{aligned}
& \int_{x_{0}}^{\tilde{x}_{0}}\left(t^{*}(x)-t_{n-1}\right)\left|\psi_{x}(x)\right| d x \\
& \quad \leq M \Delta t\left[\int_{x_{0}}^{\tilde{x}_{0}} \Delta t(x) \psi_{x}^{2}(x) d x\right]^{\frac{1}{2}}+M(\Delta t)^{\frac{5}{2}}\|\psi\|_{L^{2}\left(x_{0}, x_{1}\right)} \quad \text { if } C x \leq 1,
\end{aligned}
$$

$\int_{x_{0}}^{\tilde{x}_{0}}\left(t^{*}(x)-t_{n-1}\right)\left|\psi_{x}(x)\right| d x \leq \frac{M(\Delta t)^{\frac{3}{2}}}{(\Delta x)^{\frac{1}{2}}}\left[\int_{x_{0}}^{\tilde{x}_{0}} \Delta t(x) \psi_{x}^{2}(x) d x\right]^{\frac{1}{2}}$ if $C x>1$

Proof. We first notice that the following relations hold for any $x \in\left[x_{0}, \tilde{x}_{0}\right]$ :

$$
\begin{aligned}
& \Delta t(x)=\frac{x-x_{0}}{V\left(x, t^{*}(x)\right)}=\frac{x-x_{0}}{V\left(\tilde{x}_{0}, t_{n}\right)}+\left[\frac{x-x_{0}}{V\left(x, t^{*}(x)\right)}-\frac{x-x_{0}}{V\left(\tilde{x}_{0}, t_{n}\right)}\right] \\
& =\frac{x-x_{0}}{V\left(\tilde{x}_{0}, t_{n}\right)}+\frac{\left(x-x_{0}\right)\left[V\left(\tilde{x}_{0}, t_{n}\right)-V\left(x, t^{*}(x)\right)\right]}{V\left(x, t^{*}(x)\right) V\left(\tilde{x}_{0}, t_{n}\right)} \\
& =\frac{x-x_{0}}{V\left(\tilde{x}_{0}, t_{n}\right)}[1+\mathcal{O}(\Delta t)] \\
& =\frac{x-x_{0}}{V\left(\tilde{x}_{0}, t_{n}\right)}+\mathcal{O}\left(\Delta t\left(x-x_{0}\right)\right), \\
& t^{*}(x)-t_{n-1} \\
& =\Delta t-\Delta t(x) \\
& =\frac{\tilde{x}_{0}-x_{0}}{V\left(\tilde{x}_{0}, t_{n}\right)}-\left[\frac{x-x_{0}}{V\left(\tilde{x}_{0}, t_{n}\right)}+\frac{\left(x-x_{0}\right)\left[V\left(\tilde{x}_{0}, t_{n}\right)-V\left(x, t^{*}(x)\right)\right]}{V\left(x, t^{*}(x)\right) V\left(\tilde{x}_{0}, t_{n}\right)}\right] \\
& =\frac{\tilde{x}_{0}-x}{V\left(\tilde{x}_{0}, t_{n}\right)}+\mathcal{O}\left(\Delta t\left(x-x_{0}\right)\right) \text {. }
\end{aligned}
$$

Let $x_{i-\frac{1}{2}}(i=1,2, \ldots, I)$ be defined by

$$
x_{i-\frac{1}{2}}:=\frac{x_{i-1}+x_{i}}{2}=x_{i-1}+\frac{\Delta x}{2} .
$$

We first prove the lemma for $C x \leq 1$, i.e., the estimate (6.2). Using the inverse inequalities (3.8) and (6.4) and (6.5), we bound the left-hand side of (6.2) by

$$
\begin{aligned}
& \int_{x_{0}}^{\tilde{x}_{0}}\left(t^{*}(x)-t_{n-1}\right)\left|\psi_{x}(x)\right| d x \\
& =\left|\psi_{x}\left(x_{i-\frac{1}{2}}\right)\right| \int_{x_{0}}^{\tilde{x}_{0}}\left[\frac{\tilde{x}_{0}-x}{V\left(\tilde{x}_{0}, t_{n}\right)}+\mathcal{O}\left(\Delta t\left(x-x_{0}\right)\right)\right] d x \\
& =\left|\psi_{x}\left(x_{i-\frac{1}{2}}\right)\right|\left[\frac{\left(\tilde{x}_{0}-x_{0}\right) \Delta t}{2}+\mathcal{O}\left((\Delta t)^{3}\right)\right] \\
& =\left|\psi_{x}\left(x_{\frac{1}{2}}\right)\right| \int_{x_{0}}^{\tilde{x}_{0}}\left[\frac{x-x_{0}}{V\left(\tilde{x}_{0}, t_{n}\right)}+\mathcal{O}\left((\Delta t)^{2}\right)\right] d x \\
& =\int_{x_{0}}^{\tilde{x}_{0}}\left[\Delta t(x)+\mathcal{O}\left((\Delta t)^{2}\right)\right]\left|\psi_{x}(x)\right| d x \\
& \leq M \Delta t\left[\int_{x_{0}}^{\tilde{x}_{0}} \Delta t(x) \psi_{x}^{2}(x) d x\right]^{\frac{1}{2}}+M(\Delta t)^{\frac{5}{2}}\|\psi\|_{L^{2}\left(x_{0}, x_{1}\right)} .
\end{aligned}
$$


Thus, we prove the estimate (6.2). We now turn to the estimate (6.3). In this case, $C x>1$. We bound the left-hand side of (6.3) from above as follows:

$$
\begin{aligned}
& \int_{x_{0}}^{\tilde{x}_{0}}\left(t^{*}(x)-t_{n-1}\right)\left|\psi_{x}(x)\right| d x \\
&=\left|\psi_{x}\left(x_{I c-\frac{1}{2}}\right)\right| \int_{x_{I c-1}}^{\tilde{x}_{0}}\left(t^{*}(x)-t_{n-1}\right) d x \\
& \quad+\sum_{i=1}^{I c-1}\left|\psi_{x}\left(x_{i-\frac{1}{2}}\right)\right| \int_{x_{i-1}}^{x_{i}}\left(t^{*}(x)-t_{n-1}\right) d x \\
&=\left|\psi_{x}\left(x_{I c-\frac{1}{2}}\right)\right| \int_{x_{I c-1}}^{\tilde{x}_{0}}\left[\frac{\tilde{x}_{0}-x}{V\left(\tilde{x}_{0}, t_{n}\right)}+\mathcal{O}\left(\Delta t\left(x-x_{0}\right)\right)\right] d x \\
& \quad+\sum_{i=1}^{I c-1}\left|\psi_{x}\left(x_{i-\frac{1}{2}}\right)\right| \int_{x_{i-1}}^{x_{i}}\left[\frac{\tilde{x}_{0}-x}{V\left(\tilde{x}_{0}, t_{n}\right)}+\mathcal{O}\left(\Delta t\left(x-x_{0}\right)\right)\right] d x \\
& \leq \frac{\tilde{x}_{0}-x_{I c-1}}{V\left(\tilde{x}_{0}, t_{n}\right)}\left|\psi_{x}\left(x_{I c-\frac{1}{2}}\right)\right|\left[\frac{\tilde{x}_{0}-x_{I c-1}}{2}+M \Delta t\left(\frac{\tilde{x}_{0}+x_{I c-1}}{I c-1}-x_{0}\right)\right] \\
&+\frac{\Delta x}{V\left(\tilde{x}_{0}, t_{n}\right)} \sum_{i=1}\left|\psi_{x}\left(x_{i-\frac{1}{2}}\right)\right|\left[\left(\tilde{x}_{0}-x_{i-\frac{1}{2}}\right)+M \Delta t\left(x_{i-\frac{1}{2}}-x_{0}\right)\right] \\
& \leq \frac{\Delta t(1+M \Delta t)}{V\left(\tilde{x}_{0}, t_{n}\right)}\left[\left(\tilde{x}_{0}-x_{I c-1}\right)\left|\psi_{x}\left(x_{I c-\frac{1}{2}}\right)\right|+\sum_{i=1}^{I c-1} \Delta x\left|\psi_{x}\left(x_{i-\frac{1}{2}}\right)\right|\right] \\
&= \frac{\Delta t(1+M \Delta t)}{V\left(\tilde{x}_{0}, t_{n}\right)} \int_{x_{0}}^{\tilde{x}_{0}}\left|\psi_{x}(x)\right| d x \\
& \leq \frac{(\Delta t)^{\frac{3}{2}}(1+M \Delta t)}{V V^{\frac{1}{2}}\left(\tilde{x}_{0}, t_{n}\right)}\left\|\psi_{x}\right\|_{L^{2}\left(x_{0}, \tilde{x}_{0}\right)} .
\end{aligned}
$$

On the other hand, we bound the right-hand side of (6.3) from below by

$$
\begin{aligned}
\int_{x_{0}}^{\tilde{x}_{0}} & \Delta t(x) \psi_{x}^{2}(x) d x \\
= & \sum_{i=1}^{I c-1} \psi_{x}^{2}\left(x_{i-\frac{1}{2}}\right) \int_{x_{i-1}}^{x_{i}} \Delta t(x) d x+\psi_{x}^{2}\left(x_{I c-\frac{1}{2}}\right) \int_{x_{I c-1}}^{\tilde{x}_{0}} \Delta t(x) d x \\
\geq & (1-M \Delta t) \sum_{i=1}^{I c-1} \psi_{x}^{2}\left(x_{i-\frac{1}{2}}\right) \int_{x_{i-1}}^{x_{i}} \frac{x-x_{0}}{V\left(\tilde{x}_{0}, t_{n}\right)} d x \\
& +(1-M \Delta t) \psi_{x}^{2}\left(x_{I c-\frac{1}{2}}\right) \int_{x_{I c-1}}^{\tilde{x}_{0}} \frac{x-x_{0}}{V\left(\tilde{x}_{0}, t_{n}\right)} d x \\
\geq & \frac{(1-M \Delta t) \Delta x}{V\left(\tilde{x}_{0}, t_{n}\right)} \sum_{i=1}^{I c-1} \psi_{x}^{2}\left(x_{i-\frac{1}{2}}\right)\left(x_{i-\frac{1}{2}}-x_{0}\right) \\
& +\frac{(1-M \Delta t)\left(\tilde{x}_{0}-x_{I c-1}\right)}{V\left(\tilde{x}_{0}, t_{n}\right)} \psi_{x}^{2}\left(x_{I c-\frac{1}{2}}\right)\left(\frac{\tilde{x}_{0}+x_{I c-1}}{2}-x_{0}\right) \\
\geq & \frac{(1-M \Delta t) \Delta x}{2 V\left(\tilde{x}_{0}, t_{n}\right)}\left[\sum_{i=1}^{I c-1} \psi_{x}^{2}\left(x_{i-\frac{1}{2}}\right) \Delta x+\psi_{x}^{2}\left(x_{I c-\frac{1}{2}}\right)\left(\tilde{x}_{0}-x_{I c-1}\right)\right] \\
= & \frac{(1-M \Delta t) \Delta x}{2 V\left(\tilde{x}_{0}, t_{n}\right)}\left\|\psi_{x}\right\|_{L^{2}\left(x_{0}, \tilde{x}_{0}\right)}^{2} .
\end{aligned}
$$

Combining the estimates (6.8) and (6.9), we prove (6.3). 
LEMMA 6.2. Under the assumptions of Lemma 6.1, the estimate (6.20) holds for any piecewise-linear function $\psi(x)$ defined on $\left[x_{0}, x_{I}\right]$ with the partition $(2.4)$

$$
\begin{aligned}
& \int_{x_{0}}^{\tilde{x}_{0}}\left(t^{*}(x)-t_{n-1}\right)|\psi(x)| d x \\
& \quad \leq \frac{M(\Delta t)^{\frac{3}{2}}}{(\Delta x)^{\frac{1}{2}}}\left[\int_{x_{0}}^{x_{\max \{I c, 2\}}} \Delta t(x) \psi^{2}(x) d x\right]^{\frac{1}{2}}+M(\Delta t)^{\frac{5}{2}}\|\psi\|_{L^{2}\left(x_{0}, \tilde{x}_{0}\right)} .
\end{aligned}
$$

Proof. Using (6.5), we bound the left-hand side of (6.10) for $C x \leq 1$

$$
\begin{aligned}
\int_{x_{0}}^{\tilde{x}_{0}}\left(t^{*}(x)-t_{n-1}\right)|\psi(x)| d x & \int_{x_{0}}^{\tilde{x}_{0}}\left(t^{*}(x)-t_{n-1}\right)\left[\left|\psi\left(x_{0}\right)\right| \frac{x_{1}-x}{\Delta x}+\left|\psi\left(x_{1}\right)\right| \frac{x-x_{0}}{\Delta x}\right] d x \\
\leq & \frac{1}{V\left(\tilde{x}_{0}, t_{n}\right) \Delta x} \int_{x_{0}}^{\tilde{x}_{0}}\left(\tilde{x}_{0}-x\right)\left[\left|\psi\left(x_{0}\right)\right|\left(x_{1}-x\right)+\left|\psi\left(x_{1}\right)\right|\left(x-x_{0}\right)\right] d x \\
& +M(\Delta t)^{2} \int_{x_{0}}^{\tilde{x}_{0}}|\psi(x)| d x \\
\leq & \frac{\left(\tilde{x}_{0}-x_{0}\right)^{2}}{2 V\left(\tilde{x}_{0}, t_{n}\right)}\left[\left|\psi\left(x_{0}\right)\right|+\left|\psi\left(x_{1}\right)\right| \frac{\left(\tilde{x}_{0}-x_{0}\right)}{3 \Delta x}\right]+M(\Delta t)^{2} \int_{x_{0}}^{\tilde{x}_{0}}|\psi(x)| d x \\
\leq & M(\Delta t)^{2}\left[\psi^{2}\left(x_{0}\right)+\psi^{2}\left(x_{1}\right)\right]^{\frac{1}{2}}+M(\Delta t)^{\frac{5}{2}}\|\psi\|_{L^{2}\left(x_{0}, \tilde{x}_{0}\right)} \cdot
\end{aligned}
$$

Using (6.4), we bound the right-hand side of (6.10) from below

$$
\begin{aligned}
\int_{x_{0}}^{x_{2}} & \Delta t(x) \psi^{2}(x) d x \\
= & \int_{x_{0}}^{\tilde{x}_{0}} \Delta t(x) \psi^{2}(x) d x+\int_{\tilde{x}_{0}}^{x_{1}} \Delta t \psi^{2}(x) d x+\int_{x_{1}}^{x_{2}} \Delta t \psi^{2}(x) d x \\
\geq & \frac{1-M \Delta t}{V\left(\tilde{x}_{0}, t_{n}\right)(\Delta x)^{2}} \int_{x_{0}}^{\tilde{x}_{0}}\left(x-x_{0}\right)\left[\psi\left(x_{0}\right)\left(x_{1}-x\right)+\psi\left(x_{1}\right)\left(x-x_{0}\right)\right]^{2} d x \\
& +\frac{(1-M \Delta t) \Delta t}{(\Delta x)^{2}} \int_{\tilde{x}_{0}}^{x_{1}}\left[\psi\left(x_{0}\right)\left(x_{1}-x\right)+\psi\left(x_{1}\right)\left(x-x_{0}\right)\right]^{2} d x \\
& +\frac{(1-M \Delta t) \Delta t}{(\Delta x)^{2}} \int_{x_{1}}^{x_{2}}\left[\psi\left(x_{1}\right)\left(x_{2}-x\right)+\psi\left(x_{2}\right)\left(x-x_{1}\right)\right]^{2} d x \\
= & \frac{(1-M \Delta t) \Delta x \Delta t}{12}\left[\psi^{2}\left(x_{0}\right)\left(4-6 C x+4 C x^{2}-C x^{3}\right)\right. \\
& \left.+\psi\left(x_{0}\right) \psi\left(x_{1}\right)\left(4-4 C x^{2}+2 C x^{3}\right)+\psi^{2}\left(x_{1}\right)\left(4-C x^{3}\right)\right] \\
& +\frac{(1-M \Delta t) \Delta x \Delta t}{3}\left[\psi^{2}\left(x_{1}\right)+\psi\left(x_{1}\right) \psi\left(x_{2}\right)+\psi^{2}\left(x_{2}\right)\right] \\
\geq & \frac{(1-M \Delta t) \Delta x \Delta t}{24}\left\{\psi^{2}\left(x_{0}\right)[1+(1-C x)(1+(1-C x)(4-3 C x))]\right. \\
& \left.\left.+\psi^{2}\left(x_{1}\right)\left(6+8 C x^{2}-6 C x^{3}\right)\right]\right\} \\
\geq & \frac{(1-M \Delta t) \Delta x \Delta t}{24}\left[\psi^{2}\left(x_{0}\right)+\psi^{2}\left(x_{1}\right)\right]
\end{aligned}
$$


where we used the fact that $\Delta x /\left(V\left(\tilde{x}_{0}, t_{n}\right) \Delta t\right)=1 / C x$ at the second equality and the simple inequality

$$
a b \leq \frac{a^{2}}{4}+b^{2}
$$

repeatedly at the second " $\geq$ " sign, and the fact that $C x \leq 1$.

The combination of the estimates (6.11) and (6.12) deduces the estimate (6.10) in the context of $C x \leq 1$. We now turn to the case $C x>1$. Using (6.5), we rewrite the left-hand side of $(6.10)$ as

$$
\begin{aligned}
\int_{x_{0}}^{\tilde{x}_{0}} & \left(t^{*}(x)-t_{n-1}\right)|\psi(x)| d x \\
\leq & \sum_{i=1}^{I c-1} \int_{x_{i-1}}^{x_{i}} \frac{\tilde{x}_{0}-x}{V\left(\tilde{x}_{0}, t_{n}\right)}\left[\left|\psi\left(x_{i-1}\right)\right| \frac{x_{i}-x}{\Delta x}+\left|\psi\left(x_{i}\right)\right| \frac{x-x_{i-1}}{\Delta x}\right] d x \\
& +\int_{x_{I c-1}}^{\tilde{x}_{0}} \frac{\tilde{x}_{0}-x}{V\left(\tilde{x}_{0}, t_{n}\right)}\left[\left|\psi\left(x_{I c-1}\right)\right| \frac{x_{I c}-x}{\Delta x}+\left|\psi\left(x_{I c}\right)\right| \frac{x-x_{I c-1}}{\Delta x}\right] d x \\
& +\int_{x_{0}}^{\tilde{x}_{0}} \mathcal{O}\left(\left(x-x_{0}\right) \Delta t\right)|\psi(x)| d x .
\end{aligned}
$$

The first term on the right-hand side is bounded by

$$
\begin{aligned}
& \sum_{i=1}^{I c-1} \int_{x_{i-1}}^{x_{i}} \frac{\tilde{x}_{0}-x}{V\left(\tilde{x}_{0}, t_{n}\right)}\left[\left|\psi\left(x_{i-1}\right)\right| \frac{x_{i}-x}{\Delta x}+\left|\psi\left(x_{i}\right)\right| \frac{x-x_{i-1}}{\Delta x}\right] d x \\
& =\frac{\Delta x}{V\left(\tilde{x}_{0}, t_{n}\right)} \sum_{i=1}^{I c-1}\left[\left|\psi\left(x_{i-1}\right)\right|\left(\frac{\tilde{x}_{0}-x_{i}}{2}+\frac{\Delta x}{3}\right)\right. \\
& \left.\quad+\left|\psi\left(x_{i}\right)\right|\left(\frac{\tilde{x}_{0}-x_{i-1}}{2}-\frac{\Delta x}{3}\right)\right] \\
& \leq \frac{\sqrt{2} \Delta x}{2 V\left(\tilde{x}_{0}, t_{n}\right)} \sum_{i=1}^{I c-1}\left(\tilde{x}_{0}-x_{i-1}\right)\left[\psi^{2}\left(x_{i-1}\right)+\psi^{2}\left(x_{i}\right)\right]^{\frac{1}{2}} .
\end{aligned}
$$

The second term on the right-hand side of (6.14) has an upper bound

$$
\begin{aligned}
& \int_{x_{I c-1}}^{\tilde{x}_{0}} \frac{\tilde{x}_{0}-x}{V\left(\tilde{x}_{0}, t_{n}\right)}\left[\left|\psi\left(x_{I c-1}\right)\right| \frac{x_{I c}-x}{\Delta x}+\left|\psi\left(x_{I c}\right)\right| \frac{x-x_{I c-1}}{\Delta x}\right] d x \\
& =\frac{1}{V\left(\tilde{x}_{0}, t_{n}\right)}\left[\left|\psi\left(x_{I c-1}\right)\right|\left(\frac{\left(x_{I c}-\tilde{x}_{0}\right)\left(\tilde{x}_{0}-x_{I c-1}\right)^{2}}{2 \Delta x}+\frac{\left(\tilde{x}_{0}-x_{I c-1}\right)^{3}}{3 \Delta x}\right)\right. \\
& \left.\quad+\left|\psi\left(x_{I c}\right)\right| \frac{\left(\tilde{x}_{0}-x_{I c-1}\right)^{3}}{6 \Delta x}\right] \\
& \leq \frac{1}{2 V\left(\tilde{x}_{0}, t_{n}\right)}\left[\left|\psi\left(x_{I c-1}\right)\right|\left(\tilde{x}_{0}-x_{I c-1}\right)^{2}+\left|\psi\left(x_{I c}\right)\right|\left(\tilde{x}_{0}-x_{I c-1}\right)^{2}\right] \\
& \leq \frac{\sqrt{2} \Delta x\left(\tilde{x}_{0}-x_{I c-1}\right)}{2 V\left(\tilde{x}_{0}, t_{n}\right)}\left[\psi^{2}\left(x_{I c-1}\right)+\psi^{2}\left(x_{I c}\right)\right]^{\frac{1}{2}} .
\end{aligned}
$$

The third term on the right-hand side of (6.14) is controlled by

$$
\left|\int_{x_{0}}^{\tilde{x}_{0}} \mathcal{O}\left(\left(x-x_{0}\right) \Delta t\right)\right| \psi(x)|d x| \leq M(\Delta t)^{\frac{5}{2}}\|\psi\|_{L^{2}\left(x_{0}, \tilde{x}_{0}\right)} .
$$


Combining the estimates (6.14) through (6.17), we obtain an upper bound for the left-hand side of (6.10) in the case of $C x>1$ as follows:

$$
\begin{aligned}
& \int_{x_{0}}^{\tilde{x}_{0}}\left(t^{*}(x)-t_{n-1}\right)|\psi(x)| d x \\
& \leq \frac{\sqrt{2} \Delta x}{2 V\left(\tilde{x}_{0}, t_{n}\right)} \sum_{i=1}^{I c}\left(\tilde{x}_{0}-x_{i-1}\right)\left[\psi^{2}\left(x_{i-1}\right)+\psi^{2}\left(x_{i}\right)\right]^{\frac{1}{2}} \\
& \quad+M(\Delta t)^{\frac{5}{2}}\|\psi\|_{L^{2}\left(x_{0}, \tilde{x}_{0}\right)} .
\end{aligned}
$$

We are now in a position to bound the right-hand side of (6.10) from below in the context of $C x>1$. Using (6.4), we have

$$
\begin{aligned}
& \int_{x_{0}}^{x_{I c}} \Delta t(x) \psi^{2}(x) d x \\
& =\int_{x_{0}}^{\tilde{x}_{0}} \Delta t(x) \psi^{2}(x) d x+\int_{\tilde{x}_{0}}^{x_{I c}} \Delta t \psi^{2}(x) d x \\
& \geq \frac{1-M \Delta t}{V\left(\tilde{x}_{0}, t_{n}\right)(\Delta x)^{2}}\left\{\sum _ { i = 1 } ^ { I c - 1 } \int _ { x _ { i - 1 } } ^ { x _ { i } } ( x - x _ { 0 } ) \left[\psi\left(x_{i-1}\right)\left(x_{i}-x\right)\right.\right. \\
& \left.\quad+\psi\left(x_{i}\right)\left(x-x_{i-1}\right)\right]^{2} d x \\
& \left.\quad+\int_{x_{I c-1}}^{\tilde{x}_{0}}\left(x-x_{0}\right)\left[\psi\left(x_{I c-1}\right)\left(x_{I c}-x\right)+\psi\left(x_{I c}\right)\left(x-x_{I c-1}\right)\right]^{2} d x\right\} \\
& \quad+\frac{\Delta t}{(\Delta x)^{2}} \int_{\tilde{x}_{0}}^{x_{I c}}\left[\psi\left(x_{I c-1}\right)\left(x_{I c}-x\right)+\psi\left(x_{I c}\right)\left(x-x_{I c-1}\right)\right]^{2} d x .
\end{aligned}
$$

The first term on the right-hand side is bounded from below

$$
\begin{gathered}
\frac{1-M \Delta t}{V\left(\tilde{x}_{0}, t_{n}\right)(\Delta x)^{2}} \sum_{i=1}^{I c-1} \int_{x_{i-1}}^{x_{i}}\left(x-x_{0}\right)\left[\psi\left(x_{i-1}\right)\left(x_{i}-x\right)+\psi\left(x_{i}\right)\left(x-x_{i-1}\right)\right]^{2} d x \\
=\frac{(1-M \Delta t)(\Delta x)^{2}}{12 V\left(\tilde{x}_{0}, t_{n}\right)} \sum_{i=1}^{I c-1}\left[(4 i-3) \psi^{2}\left(x_{i-1}\right)+(2 i-1) \psi\left(x_{i-1}\right) \psi\left(x_{i}\right)\right. \\
\left.\quad+(4 i-1) \psi^{2}\left(x_{i}\right)\right] \\
\geq \frac{(1-M \Delta t)(\Delta x)^{2}}{12 V\left(\tilde{x}_{0}, t_{n}\right)} \sum_{i=1}^{I c-1}\left[\left(\frac{i}{2}+3(i-1)\right) \psi^{2}\left(x_{i-1}\right)+2 i \psi^{2}\left(x_{i}\right)\right] \\
\geq \frac{(1-M \Delta t)(\Delta x)^{2}}{24 V\left(\tilde{x}_{0}, t_{n}\right)} \sum_{i=1}^{I c-1} i\left[\psi^{2}\left(x_{i-1}\right)+\psi^{2}\left(x_{i}\right)\right],
\end{gathered}
$$

where we used the simple inequality (6.13) again.

Let $\alpha=\left(\tilde{x}_{0}-x_{I c-1}\right) / \Delta x$, we evaluate the second and the third terms on the right-hand side of (6.19), respectively, 


$$
\begin{aligned}
& \frac{1-M \Delta t}{V\left(\tilde{x}_{0}, t_{n}\right)(\Delta x)^{2}} \int_{x_{I c-1}}^{\tilde{x}_{0}}\left(x-x_{0}\right)\left[\psi\left(x_{I c-1}\right)\left(x_{I c}-x\right)+\psi\left(x_{I c}\right)\left(x-x_{I c-1}\right)\right]^{2} d x \\
& =\frac{(1-M \Delta t)(\Delta x)^{2}}{12 V\left(\tilde{x}_{0}, t_{n}\right)}\left[\left[4 I c\left(1-(1-\alpha)^{3}\right)+3(1-\alpha)^{4}-3\right] \psi^{2}\left(x_{I c-1}\right)\right. \\
& +\left[12(I c-1) \alpha^{2}-8(I c-2) \alpha^{3}-6 \alpha^{4}\right] \psi\left(x_{I c-1}\right) \psi\left(x_{I c}\right) \\
& \left.+\left[4(I c-1) \alpha^{3}+3 \alpha^{4}\right] \psi^{2}\left(x_{I c}\right)\right]^{2} \\
& \frac{\Delta t}{(\Delta x)^{2}} \int_{\tilde{x}_{0}}^{x_{I c}}\left[\psi\left(x_{I c-1}\right)\left(x_{I c}-x\right)+\psi\left(x_{I c}\right)\left(x-x_{I c-1}\right)\right]^{2} d x \\
& =\frac{\Delta x \Delta t}{3}\left[(1-\alpha)^{3} \psi^{2}\left(x_{I c-1}\right)+\left(1-3 \alpha^{2}+2 \alpha^{3}\right) \psi\left(x_{I c-1}\right) \psi\left(x_{I c}\right)\right. \\
& \left.\quad+\left(1-\alpha^{3}\right) \psi^{2}\left(x_{I c}\right)\right] \\
& \quad \frac{(\Delta x)^{2}}{12 V\left(\tilde{x}_{0}, t_{n}\right)} 4(I c-1+\alpha)\left[(1-\alpha)^{3} \psi^{2}\left(x_{I c-1}\right)\right. \\
& \left.\quad+\left(1-3 \alpha^{2}+2 \alpha^{3}\right) \psi\left(x_{I c-1}\right) \psi\left(x_{I c}\right)+\left(1-\alpha^{3}\right) \psi^{2}\left(x_{I c}\right)\right]
\end{aligned}
$$

where at the second equality we used the relation $V\left(\tilde{x}_{0}, t_{n}\right) \Delta t / \Delta x=C x=(I c-1)+\alpha$ to make the right-hand side of (6.22) consistent with that of (6.21).

The combination of (6.21) and (6.22) yields the following lower bound for the second and third terms on the right-hand side of (6.19):

$$
\begin{aligned}
& \frac{1-M \Delta t}{V\left(\tilde{x}_{0}, t_{n}\right)(\Delta x)^{2}} \int_{x_{I c-1}}^{\tilde{x}_{0}}\left(x-x_{0}\right)\left[\psi\left(x_{I c-1}\right)\left(x_{I c}-x\right)+\psi\left(x_{I c}\right)\left(x-x_{I c-1}\right)\right]^{2} d x \\
& \quad+\frac{\Delta t}{(\Delta x)^{2}} \int_{\tilde{x}_{0}}^{x_{I c}}\left[\psi\left(x_{I c-1}\right)\left(x_{I c}-x\right)+\psi\left(x_{I c}\right)\left(x-x_{I c-1}\right)\right]^{2} d x \\
& \geq \frac{(1-M \Delta t)(\Delta x)^{2}}{12 V\left(\tilde{x}_{0}, t_{n}\right)}\left[\left(4 I c-3-(1-\alpha)^{4}\right) \psi^{2}\left(x_{I c-1}\right)\right. \\
& \quad+\left(4(I c-1+\alpha)-4 \alpha^{3}+2 \alpha^{4}\right) \psi\left(x_{I c-1}\right) \psi\left(x_{I c}\right) \\
& \left.\quad+\left(4(I c-1+\alpha)-\alpha^{4}\right) \psi^{2}\left(x_{I c}\right)\right] \\
& \geq \frac{(1-M \Delta t)(\Delta x)^{2}}{12 V\left(\tilde{x}_{0}, t_{n}\right)}\left[\left(2(I c-1+\alpha)+2 \alpha(1-\alpha)^{3}\right) \psi^{2}\left(x_{I c-1}\right)\right. \\
& \left.\quad+2\left(I c-1+\alpha+\alpha^{3}-\alpha^{4}\right) \psi^{2}\left(x_{I c}\right)\right] \\
& \geq \frac{(1-M \Delta t) I c(\Delta x)^{2}}{12 V\left(\tilde{x}_{0}, t_{n}\right)}\left[\psi^{2}\left(x_{I c-1}\right)+\psi^{2}\left(x_{I c}\right)\right] .
\end{aligned}
$$

Combining the estimates (6.19), (6.20), and (6.23), we obtain

$$
\int_{x_{0}}^{x_{I c}} \Delta t(x) \psi^{2}(x) d x \geq \frac{(1-M \Delta t)(\Delta x)^{2}}{24 V\left(\tilde{x}_{0}, t_{n}\right)} \sum_{i=1}^{I c} i\left[\psi^{2}\left(x_{i-1}\right)+\psi^{2}\left(x_{i}\right)\right] .
$$


Using the estimates (6.18), (6.24), and the fact $I c \leq 2 C r$ for $C r>1$, we have

$$
\begin{aligned}
& \int_{x_{0}}^{\tilde{x}_{0}}\left(t^{*}(x)-t_{n-1}\right)|\psi(x)| d x \\
& \quad \leq \frac{\Delta x \Delta t}{V\left(\tilde{x}_{0}, t_{n}\right)} \sum_{i=1}^{I c}\left[\psi^{2}\left(x_{i-1}\right)+\psi^{2}\left(x_{i}\right)\right]^{\frac{1}{2}}+M(\Delta t)^{\frac{5}{2}}\|\psi\|_{L^{2}\left(x_{0}, \tilde{x}_{0}\right)} \\
& \quad \leq \frac{M \Delta x \Delta t}{V\left(\tilde{x}_{0}, t_{n}\right)}\left[I c \sum_{i=1}^{I c}\left[\psi^{2}\left(x_{i-1}\right)+\psi^{2}\left(x_{i}\right)\right]\right]^{\frac{1}{2}}+M(\Delta t)^{\frac{5}{2}}\|\psi\|_{L^{2}\left(x_{0}, \tilde{x}_{0}\right)} \\
& \quad \leq \frac{M(\Delta x)^{\frac{1}{2}}(\Delta t)^{\frac{3}{2}}}{V^{\frac{1}{2}}\left(\tilde{x}_{0}, t_{n}\right)}\left[\sum_{i=1}^{I c}\left[\psi^{2}\left(x_{i-1}\right)+\psi^{2}\left(x_{i}\right)\right]\right]^{\frac{1}{2}}+M(\Delta t)^{\frac{5}{2}}\|\psi\|_{L^{2}\left(x_{0}, \tilde{x}_{0}\right)} \\
& \quad \leq M(\Delta t)^{\frac{3}{2}}(\Delta x)^{-\frac{1}{2}}\left[\int_{x_{0}}^{x_{I c}} \Delta t(x) \psi^{2}(x) d x\right]^{\frac{1}{2}}+M(\Delta t)^{\frac{5}{2}}\|\psi\|_{L^{2}\left(x_{0}, \tilde{x}_{0}\right)}
\end{aligned}
$$

Thus, we finish the proof of (6.10) for the case of $C x>1$.

LEMMA 6.3. Under Assumption (A), the following estimate holds for the fourth term on the right-hand side of (4.2):

$$
\begin{aligned}
& \left|\int_{\Omega} \Delta t(\mathbf{x}) D\left(\mathbf{x}, t_{n}\right) \nabla \xi\left(\mathbf{x}, t_{n}\right) \cdot \nabla \eta\left(\mathbf{x}, t_{n}\right) d \mathbf{x}\right| \\
& \quad \leq \varepsilon \int_{\Omega} \Delta t(\mathbf{x}) D\left(\mathbf{x}, t_{n}\right)\left|\nabla \xi\left(\mathbf{x}, t_{n}\right)\right|^{2} d \mathbf{x}+M \Delta t\left\|\xi\left(\mathbf{x}, t_{n}\right)\right\|_{L^{2}(\Omega)}^{2} \\
& \quad+M \Delta t\left[h^{4}+(\Delta t)^{2}\right]\|c\|_{L^{\infty}\left(0, T ; W^{2, \infty}(\Omega)\right)}^{2}
\end{aligned}
$$

Proof. Using (3.6) and the fact that $\Delta t(\mathbf{x})=\Delta t-\left(t^{*}(\mathbf{x})-t_{n-1}\right)$ in which $\left(t^{*}(\mathbf{x})-t_{n-1}\right)=0$ for $\mathbf{x} \in \Omega \backslash \Omega^{(I)}\left(t_{n}\right)$, we rewrite the fourth term on the right-hand side of (4.2) as follows:

$$
\begin{aligned}
\int_{\Omega} & \Delta t(\mathbf{x}) D\left(\mathbf{x}, t_{n}\right) \nabla \xi\left(\mathbf{x}, t_{n}\right) \cdot \nabla \eta\left(\mathbf{x}, t_{n}\right) d \mathbf{x} \\
= & \Delta t \int_{\Omega} D\left(\mathbf{x}, t_{n}\right) \nabla \xi\left(\mathbf{x}, t_{n}\right) \cdot \nabla \eta\left(\mathbf{x}, t_{n}\right) d \mathbf{x} \\
& -\int_{\Omega}\left(t^{*}(\mathbf{x})-t_{n-1}\right) D\left(\mathbf{x}, t_{n}\right) \nabla \xi\left(\mathbf{x}, t_{n}\right) \cdot \nabla \eta\left(\mathbf{x}, t_{n}\right) d \mathbf{x} \\
= & -\int_{\Omega^{(I)}\left(t_{n}\right)}\left(t^{*}(\mathbf{x})-t_{n-1}\right) D\left(\mathbf{x}, t_{n}\right) \nabla \xi\left(\mathbf{x}, t_{n}\right) \cdot \nabla \eta\left(\mathbf{x}, t_{n}\right) d \mathbf{x} .
\end{aligned}
$$

Without loss of generality, we assume again that the inflow and the outflow boundaries are given by (5.2). Let $I c=\lceil C r\rceil$ (i.e., the smallest integer that is larger than or equal to $C r)$. Let $\tilde{\mathbf{x}}_{0}=\left(\tilde{x}_{0}, \tilde{y}_{0}\right)$ be defined by $(4.4)$, which will backtrack to the point $\mathbf{x}_{0}=\left(x_{0}, y_{0}\right)$ at time $t_{n-1}$. We decompose this term as the sum of the following terms: 


$$
\begin{aligned}
& \left|\int_{\Omega^{(I)}\left(t_{n}\right)}\left(t^{*}(\mathbf{x})-t_{n-1}\right) D\left(\mathbf{x}, t_{n}\right) \nabla \xi\left(\mathbf{x}, t_{n}\right) \cdot \nabla \eta\left(\mathbf{x}, t_{n}\right) d \mathbf{x}\right| \\
& \leq\left|\int_{\tilde{y}_{0}}^{y_{J}} \int_{x_{0}}^{\tilde{x}_{0}(y)}\left(t^{*}(\mathbf{x})-t_{n-1}\right) D\left(\mathbf{x}, t_{n}\right) \nabla \xi\left(\mathbf{x}, t_{n}\right) \cdot \nabla \eta\left(\mathbf{x}, t_{n}\right) d \mathbf{x}\right| \\
& \quad+\int_{y_{0}}^{\tilde{y}_{0}(x)} \int_{x_{0}}^{\tilde{x}_{0}}\left(t^{*}(\mathbf{x})-t_{n-1}\right) D\left(\mathbf{x}, t_{n}\right)\left|\nabla \xi\left(\mathbf{x}, t_{n}\right)\right|\left|\nabla \eta\left(\mathbf{x}, t_{n}\right)\right| d \mathbf{x} \\
& \quad+\left|\int_{\tilde{x}_{0}}^{x_{I}} \int_{y_{0}}^{\tilde{y}_{0}(x)}\left(t^{*}(\mathbf{x})-t_{n-1}\right) D\left(\mathbf{x}, t_{n}\right) \nabla \xi\left(\mathbf{x}, t_{n}\right) \cdot \nabla \eta\left(\mathbf{x}, t_{n}\right) d \mathbf{x}\right|
\end{aligned}
$$

where $\tilde{x}_{0}(y)$ is defined in (5.11) and $\tilde{y}_{0}(x)$ is defined by symmetry.

We now estimate the first term on the right-hand side of (6.28)

$$
\begin{aligned}
\int_{\tilde{y}_{0}}^{y_{J}} & \int_{x_{0}}^{\tilde{x}_{0}(y)}\left(t^{*}(\mathbf{x})-t_{n-1}\right) D\left(\mathbf{x}, t_{n}\right) \nabla \xi\left(\mathbf{x}, t_{n}\right) \cdot \nabla \eta\left(\mathbf{x}, t_{n}\right) d \mathbf{x} \\
= & \int_{\tilde{y}_{0}}^{y_{J}} \int_{x_{0}}^{\tilde{x}_{0}(y)}\left(t^{*}(\mathbf{x})-t_{n-1}\right) D\left(\mathbf{x}, t_{n}\right) \xi_{x}\left(\mathbf{x}, t_{n}\right) \eta_{x}\left(\mathbf{x}, t_{n}\right) d \mathbf{x} \\
& +\int_{\tilde{y}_{0}}^{y_{J}} \int_{x_{0}}^{\tilde{x}_{0}(y)}\left(t^{*}(\mathbf{x})-t_{n-1}\right) D\left(\mathbf{x}, t_{n}\right) \xi_{y}\left(\mathbf{x}, t_{n}\right) \eta_{y}\left(\mathbf{x}, t_{n}\right) d \mathbf{x} .
\end{aligned}
$$

Using the last estimate in (3.7) with $k=1$, we bound the first term on the right-hand side of (6.29) by

$$
\begin{aligned}
& \left|\int_{\tilde{y}_{0}}^{y_{J}} \int_{x_{0}}^{\tilde{x}_{0}(y)}\left(t^{*}(\mathbf{x})-t_{n-1}\right) D\left(\mathbf{x}, t_{n}\right) \xi_{x}\left(\mathbf{x}, t_{n}\right) \eta_{x}\left(\mathbf{x}, t_{n}\right) d \mathbf{x}\right| \\
& \quad \leq M\left\|\eta_{x}\left(\mathbf{x}, t_{n}\right)\right\|_{L^{\infty}(\Omega)} \int_{\tilde{y}_{0}}^{y_{J}} \int_{x_{0}}^{\tilde{x}_{0}(y)}\left(t^{*}(\mathbf{x})-t_{n-1}\right)\left|\xi_{x}\left(x, y, t_{n}\right)\right| d x d y \\
& \quad \leq M h\|c\|_{L^{\infty}\left(0, T ; W^{2, \infty}(\Omega)\right)} \int_{\tilde{y}_{0}}^{y_{J}} \int_{x_{0}}^{\tilde{x}_{0}(y)}\left(t^{*}(\mathbf{x})-t_{n-1}\right)\left|\xi_{x}\left(x, y, t_{n}\right)\right| d x d y .
\end{aligned}
$$

Since $\xi\left(x, y, t_{n}\right)$ is a piecewise-linear function of $x$ for any fixed $y$, we can use Lemma 6.1 to bound the inner integral on the right-hand side of (6.30). If $\tilde{x}_{0}(y) \leq x_{1}$, the estimate (6.2) in Lemma 6.1 applies, leading to the estimate

$$
\begin{aligned}
h\|c\|_{L^{\infty}\left(0, T ; W^{2, \infty}(\Omega)\right)} \int_{x_{0}}^{\tilde{x}_{0}(y)}\left(t^{*}(\mathbf{x})-t_{n-1}\right)\left|\xi_{x}\left(x, y, t_{n}\right)\right| d x \\
\leq M \Delta t h\|c\|_{L^{\infty}\left(0, T ; W^{2, \infty}(\Omega)\right)}\left[\int_{x_{0}}^{\tilde{x}_{0}(y)} \Delta t(\mathbf{x}) \xi_{x}^{2}\left(\mathbf{x}, t_{n}\right) d x\right]^{\frac{1}{2}} \\
\quad+M(\Delta t)^{\frac{5}{2}} h\left[\int_{x_{0}}^{\tilde{x}_{0}(y)} \xi^{2}\left(\mathbf{x}, t_{n}\right) d x\right]^{\frac{1}{2}} \\
\leq \varepsilon \int_{x_{0}}^{\tilde{x}_{0}(y)} \Delta t(\mathbf{x}) \xi_{x}^{2}\left(\mathbf{x}, t_{n}\right) d x+M \Delta t \int_{x_{0}}^{\tilde{x}_{0}(y)} \xi^{2}\left(\mathbf{x}, t_{n}\right) d x \\
\quad+M(\Delta t)^{2} h^{2}\|c\|_{L^{\infty}\left(0, T ; W^{2, \infty}(\Omega)\right)}^{2} .
\end{aligned}
$$


For $\tilde{x}_{0}(y)>x_{1}$, we apply the estimate (6.3) in Lemma 6.1 and the assumption (2.5) to generate the following upper bound:

$$
\begin{aligned}
& h\|c\|_{L^{\infty}\left(0, T ; W^{2, \infty}(\Omega)\right)} \int_{x_{0}}^{\tilde{x}_{0}(y)}\left(t^{*}(\mathbf{x})-t_{n-1}\right)\left|\xi_{x}\left(x, y, t_{n}\right)\right| d x \\
& \quad \leq M(\Delta t)^{\frac{3}{2}} h^{\frac{1}{2}}\|c\|_{L^{\infty}\left(0, T ; W^{2, \infty}(\Omega)\right)}\left[\int_{x_{0}}^{\tilde{x}_{0}(y)} \Delta t(\mathbf{x}) \xi_{x}^{2}\left(\mathbf{x}, t_{n}\right) d x\right]^{\frac{1}{2}} \\
& \quad \leq \varepsilon \int_{x_{0}}^{\tilde{x}_{0}(y)} \Delta t(\mathbf{x}) \xi_{x}^{2}\left(\mathbf{x}, t_{n}\right) d x+M(\Delta t)^{3} h\|c\|_{L^{\infty}\left(0, T ; W^{2, \infty}(\Omega)\right)}^{2} .
\end{aligned}
$$

Combining the estimates (6.30)-(6.32), we come up with the following upper bound for the first term on the right-hand side of $(6.29)$ :

$$
\begin{aligned}
& \left|\int_{\tilde{y}_{0}}^{y_{J}} \int_{x_{0}}^{\tilde{x}_{0}(y)}\left(t^{*}(\mathbf{x})-t_{n-1}\right) D\left(\mathbf{x}, t_{n}\right) \xi_{x}\left(\mathbf{x}, t_{n}\right) \eta_{x}\left(\mathbf{x}, t_{n}\right) d \mathbf{x}\right| \\
& \leq \varepsilon \int_{\Omega} \Delta t(\mathbf{x}) D\left(\mathbf{x}, t_{n}\right) \xi_{x}^{2}\left(\mathbf{x}, t_{n}\right) d \mathbf{x}+M \Delta t\left\|\xi\left(\mathbf{x}, t_{n}\right)\right\|_{L^{2}(\Omega)}^{2} \\
& \quad+M \Delta t\left(h^{4}+(\Delta t)^{2}\right)\|c\|_{L^{\infty}\left(0, T ; W^{2, \infty}(\Omega)\right)}^{2}
\end{aligned}
$$

Since $\xi\left(x, y, t_{n}\right)$ is a piecewise-bilinear function of $x$ and $y, \xi_{y}\left(x, y, t_{n}\right)$ is a piecewiselinear function of $x$ for any fixed $y$. Thus, we can apply Lemma 6.2 to bound the second term on the right-hand side of $(6.29)$ by

$$
\begin{aligned}
& \left|\int_{\tilde{y}_{0}}^{y_{J}} \int_{x_{0}}^{\tilde{x}_{0}(y)}\left(t^{*}(\mathbf{x})-t_{n-1}\right) D\left(\mathbf{x}, t_{n}\right) \xi_{y}\left(\mathbf{x}, t_{n}\right) \eta_{y}\left(\mathbf{x}, t_{n}\right) d \mathbf{x}\right| \\
& \leq M\left\|\eta_{y}\left(\mathbf{x}, t_{n}\right)\right\|_{L^{\infty}(\Omega)} \int_{\tilde{y}_{0}}^{y_{J}} \int_{x_{0}}^{\tilde{x}_{0}(y)}\left(t^{*}(\mathbf{x})-t_{n-1}\right)\left|\xi_{y}\left(x, y, t_{n}\right)\right| d x d y \\
& \leq M h\|c\|_{L^{\infty}\left(0, T ; W^{2, \infty}(\Omega)\right)} \int_{\tilde{y}_{0}}^{y_{J}} \int_{x_{0}}^{\tilde{x}_{0}(y)}\left(t^{*}(\mathbf{x})-t_{n-1}\right)\left|\xi_{y}\left(x, y, t_{n}\right)\right| d x d y \\
& \leq M(\Delta t)^{\frac{3}{2}} h^{\frac{1}{2}}\|c\|_{L^{\infty}\left(0, T ; W^{2, \infty}(\Omega)\right)}\left[\int_{\Omega} \Delta t(\mathbf{x}) \xi_{y}^{2}\left(\mathbf{x}, t_{n}\right) d \mathbf{x}\right]^{\frac{1}{2}} \\
& \quad+M(\Delta t)^{\frac{5}{2}} h\|c\|_{L^{\infty}\left(0, T ; W^{2, \infty}(\Omega)\right)}\left\|\xi\left(\mathbf{x}, t_{n}\right)\right\|_{L^{2}(\Omega)} \\
& \leq \varepsilon \int_{\Omega} \Delta t(\mathbf{x}) D\left(\mathbf{x}, t_{n}\right) \xi_{y}^{2}\left(\mathbf{x}, t_{n}\right) d \mathbf{x}+M \Delta t\left\|\xi\left(\mathbf{x}, t_{n}\right)\right\|_{L^{2}(\Omega)}^{2} \\
& \quad+M(\Delta t)^{3} h\|c\|_{L^{\infty}\left(0, T ; W^{2, \infty}(\Omega)\right)}^{2}
\end{aligned}
$$

Using the estimates (6.33) and (6.34), we obtain the following upper bound for the first term on the right-hand side of (6.28):

$$
\begin{aligned}
& \left|\int_{\tilde{y}_{0}}^{y_{J}} \int_{x_{0}}^{\tilde{x}_{0}(y)}\left(t^{*}(\mathbf{x})-t_{n-1}\right) D\left(\mathbf{x}, t_{n}\right) \nabla \xi\left(\mathbf{x}, t_{n}\right) \cdot \nabla \eta\left(\mathbf{x}, t_{n}\right) d \mathbf{x}\right| \\
& \leq \varepsilon \int_{\Omega} \Delta t(\mathbf{x}) D\left(\mathbf{x}, t_{n}\right)\left|\nabla \xi\left(\mathbf{x}, t_{n}\right)\right|^{2} d \mathbf{x}+M \Delta t\left\|\xi\left(\mathbf{x}, t_{n}\right)\right\|_{L^{2}(\Omega)}^{2} \\
& \quad+M \Delta t\left[h^{4}+(\Delta t)^{2}\right]\|c\|_{L^{\infty}\left(0, T ; W^{2, \infty}(\Omega)\right)}^{2} .
\end{aligned}
$$


By symmetry, the same estimate holds for the last term on the right-hand side of (6.28). We now turn to the second term on its right-hand side. We use the estimate (3.7) and the inverse estimate (3.8) to derive an upper bound for this term

$$
\begin{aligned}
& \int_{y_{0}}^{\tilde{y}_{0}(x)} \int_{x_{0}}^{\tilde{x}_{0}}\left(t^{*}(\mathbf{x})-t_{n-1}\right) D\left(\mathbf{x}, t_{n}\right)\left|\nabla \xi\left(\mathbf{x}, t_{n}\right)\right|\left|\nabla \eta\left(\mathbf{x}, t_{n}\right)\right| d \mathbf{x} \\
& \quad \leq M \Delta t\left\|\eta\left(\mathbf{x}, t_{n}\right)\right\|_{W^{1, \infty}(\Omega)} \int_{y_{0}}^{\tilde{y}_{0}(x)} \int_{x_{0}}^{\tilde{x}_{0}}\left|\nabla \xi\left(\mathbf{x}, t_{n}\right)\right| d \mathbf{x} \\
& \quad \leq M(\Delta t)^{2} h\|c\|_{L^{\infty}\left(0, T ; W^{2, \infty}(\Omega)\right)}\left[\int_{y_{0}}^{\tilde{y}_{0}(x)} \int_{x_{0}}^{\tilde{x}_{0}}\left|\nabla \xi\left(\mathbf{x}, t_{n}\right)\right|^{2} d \mathbf{x}\right]^{\frac{1}{2}} \\
& \quad \leq M(\Delta t)^{2}\|c\|_{L^{\infty}\left(0, T ; W^{2, \infty}(\Omega)\right)}\left\|\xi\left(\mathbf{x}, t_{n}\right)\right\|_{L^{2}(\Omega)} \\
& \quad \leq M \Delta t\left\|\xi\left(\mathbf{x}, t_{n}\right)\right\|_{L^{2}(\Omega)}^{2}+M(\Delta t)^{3}\|c\|_{L^{\infty}\left(0, T ; W^{2, \infty}(\Omega)\right)}^{2} .
\end{aligned}
$$

Thus, combining (6.27) and (6.28), (6.35), and (6.36), we prove the lemma.

7. Numerical experiments. In this section we present numerical experiments to verify the theoretically proven optimal-order $L^{2}$ convergence rates. The example considered is the transport of a two-dimensional rotating Gaussian pulse. The spatial domain is $\Omega=(-0.5,0.5) \times(-0.5,0.5)$, the rotating field is imposed as $V^{(1)}(x, y)=$ $-4 y$, and $V^{(2)}(x, y)=4 x$. The time interval is $[0, T]=[0, \pi / 2]$, which is the time period required for one complete rotation. The initial condition $c_{0}(x, y)$ is given by

$$
c_{0}(x, y):=\exp \left(-\frac{\left(x-x_{c}\right)^{2}+\left(y-y_{c}\right)^{2}}{2 \sigma^{2}}\right)
$$

where $x_{c}, y_{c}$, and $\sigma$ are the centered and standard deviations, respectively. The corresponding analytical solution for (2.1) with $R=1$, a constant diffusion coefficient $D$, and $f=0$ is given by

$$
u(x, y, t)=\frac{2 \sigma^{2}}{2 \sigma^{2}+4 D t} \exp \left(-\frac{\left(\bar{x}-x_{c}\right)^{2}+\left(\bar{y}-y_{c}\right)^{2}}{2 \sigma^{2}+4 D t}\right),
$$

where $\bar{x}=x \cos (4 t)+y \sin (4 t)$ and $\bar{y}=-x \sin (4 t)+y \cos (4 t)$.

In the numerical experiments, the data are chosen as follows: $D=10^{-4}, x_{c}=$ $-0.25, y_{c}=0, \sigma=0.0447$ which gives $2 \sigma^{2}=0.0040$. This problem provides an example for a homogeneous two-dimensional advection-diffusion equation with a variable velocity field and a known analytical solution. This example has been used widely to test for numerical artifacts of different schemes, such as numerical stability and numerical dispersion, spurious oscillations, and phase errors. Our previous work $[30,31]$ showed that the ELLAM scheme yields accurate numerical solutions that are free of numerical diffusion, spurious oscillation, and phase errors, even though large time steps are used. It also showed that the ELLAM scheme often outperforms many well received and widely used numerical methods.

In this section we use a linear regression to fit the convergence rates and the associated constants in the error estimates

$$
\|C(\mathbf{x}, T)-c(\mathbf{x}, T)\|_{L^{2}(\Omega)} \leq M_{\alpha} h^{\alpha}+M_{\beta}(\Delta t)^{\beta} .
$$

We perform two kinds of computations. The first tests the spatial convergence rate of the ELLAM scheme, where we fix a small time step $\Delta t$ and compute the constant 


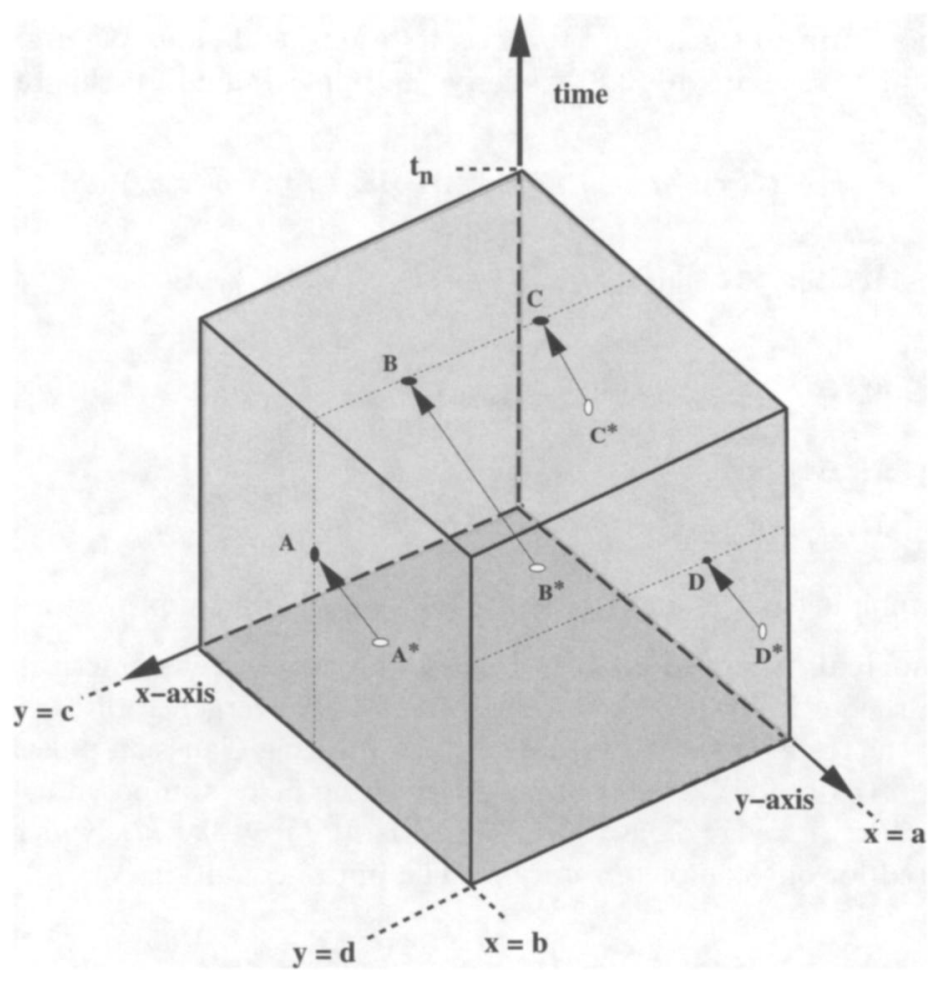

FIG. 7.1.

TABLE 7.1

Convergence rates in space, test for $M_{\alpha}$ and $\alpha$.

\begin{tabular}{|c|c|c|}
\hline$h$ & $\Delta t$ & $\|C(\mathbf{x}, T)-c(\mathbf{x}, T)\|_{L^{2}(\Omega)}$ \\
\hline$\pi / 120$ & $1 / 16$ & $3.567925 \times 10^{-2}$ \\
\hline$\pi / 120$ & $1 / 32$ & $9.562615 \times 10^{-3}$ \\
\hline$\pi / 120$ & $1 / 48$ & $3.594445 \times 10^{-3}$ \\
\hline$\pi / 120$ & $1 / 64$ & $1.991497 \times 10^{-3}$ \\
\hline & & $M_{\alpha}=12.4041, \alpha=2.0957$ \\
\hline
\end{tabular}

$M_{\alpha}$ and the rate $\alpha$ with respect to $h$; the other tests the temporal convergence rate, where we choose a small grid size $h$ and calculate the constant $M_{\beta}$ and the rate $\beta$ with respect to $\Delta t$. The results are presented in Tables 7.1 and 7.2 , fitting the pairs $\left(M_{\alpha}, \alpha\right)$ and $\left(M_{\beta}, \beta\right)$, respectively. They show that the ELLAM scheme possesses second-order accuracy in space and first-order accuracy in time as predicted by the theorem in section 3 . Moreover we notice that the numerical experiments $M_{\beta}$ is much smaller than $M_{\alpha}$. This reflects the fact that the solutions of the equation (2.1) are not smooth in space but are much smoother along characteristics, and justifies the use of the Lagrangian coordinates in the ELLAM schemes (see Figure 7.1). 
TABLE 7.2

Convergence rates in time, test for $M_{\beta}$ and $\beta$.

\begin{tabular}{|c|c|c|}
\hline$\Delta t$ & $h$ & $\|C(\mathbf{x}, T)-c(\mathbf{x}, T)\|_{L^{2}(\Omega)}$ \\
\hline$\pi / 20$ & $1 / 64$ & $1.407027 \times 10^{-2}$ \\
\hline$\pi / 40$ & $1 / 64$ & $7.387595 \times 10^{-3}$ \\
\hline$\pi / 60$ & $1 / 64$ & $4.262668 \times 10^{-3}$ \\
\hline$\pi / 80$ & $1 / 64$ & $2.964727 \times 10^{-3}$ \\
\hline & & $M_{\beta}=0.1181, \beta=1.1253$ \\
\hline
\end{tabular}

\section{REFERENCES}

[1] T. Arbogast AND M. F. WheELER, A characteristic-mixed finite element method for advection-dominated transport problems, SIAM J. Numer. Anal., 32 (1995), pp. 404-424.

[2] J. W. Barrett AND K. W. Morton, Approximate symmetrization and Petrov-Galerkin methods for diffusion-convection problems, Comput. Methods Appl. Mech. Engrg., 45 (1984), pp. $97-122$.

[3] J. BeAR, Hydraulics of Groundwater, McGraw-Hill, New York, 1979.

[4] E. T. Bouloutas And M. A. Celia, An improved cubic Petrov-Galerkin method for simulation of transient advection-diffusion processes in rectangularly decomposable domains, Comput. Methods Appl. Mech. Engrg., 91 (1991), pp. 289-308.

[5] A. Brooks And T. J. R. Hughes, Streamline upwind Petrov-Galerkin formulations for convection dominated flows with particular emphasis on the incompressible Navier-Stokes equations, Comput. Methods Appl. Mech. Engrg., 32 (1982), pp. 199-259.

[6] M. A. Celia, T. F. Russell, I. Herrera, And R. E. Ewing, An Eulerian-Lagrangian localized adjoint method for the advection-diffusion equation, Adv. in Water Res., 13 (1990), pp. 187-206.

[7] I. Christie, D. F. Griffiths, A. R. Mitchell, And O. C. Zienkiewicz, Finite element methods for second order differential equations with significant first derivatives, Internat. J. Numer. Engrg., 10 (1976), pp. 1389-1396.

[8] P. G. Ciarlet And J. L. Lions, Eds., Handbook of Numerical Analysis, Vol. 2, Finite Element Methods (Part 1), North-Holland, Amsterdam, 1991.

[9] P. Colella, A direct Eulerian MUSCL scheme for gas dynamics, SIAM J. Sci. Stat. Comput., 6 (1985), pp. 104-117.

[10] M. G. CRANDAll AND A. MAJDA, Monotone difference approximations for scalar conservation laws, Math. Comp., 34 (1980), pp. 1-21.

[11] J. Douglas, JR. AND T. F. Russell, Numerical methods for convection-dominated diffusion problems based on combining the method of characteristics with finite element or finite difference procedures, SIAM J. Numer. Anal., 19 (1982), pp. 871-885.

[12] B. Einfeldt, On Godunov-type methods for gas dynamics, SIAM J. Numer. Anal., 25 (1988), pp. 294-318.

[13] R. E. EwIng, ED., The Mathematics of Reservoir Simulation, Frontiers Appl. Math. 1, SIAM, Philadelphia, 1984.

[14] B. A. Finlayson, Numerical Methods for Problems with Moving Fronts, Ravenna Park Publishing, Seattle, 1992.

[15] A. Harten, B. Engquist, S. Osher, And S. Chakravarthy, Uniformly high order accurate essentially nonoscillatory schemes, III, J. Comput. Phys., 71 (1987), pp. 231-303.

[16] I. Herrera, R. E. Ewing, M. A. Celia, And T. F. Russell, Eulerian-Lagrangian localized adjoint methods: The theoretical framework, Numer. Methods Partial Differential Equations, 9 (1993), pp. 431-458.

[17] T. J. R. Hughes And M. Mallet, A new finite element formulation for computational fluid dynamics: III. The general streamline operator for multidimensional advective-diffusive systems, Comput. Methods Appl. Mech. Engrg., 58 (1986), pp. 305-328.

[18] C. Johnson, A. SzePESSY, AND P. HANSBO, On the convergence of shock-capturing streamline diffusion finite element methods for hyperbolic conservation laws, Math. Comp., 54 (1990), pp. 107-129. 
[19] K. W. Morton, A. Priestley, AND E. SÜLI, Stability of the Lagrangian-Galerkin method with nonexact integration, RAIRO Modél. Math. Anal. Numer., 22 (1988), pp. 625-653.

[20] O. Pironneau, On the transport-diffusion algorithm and its application to the Navier-Stokes equations, Numer. Math., 38 (1982), pp. 309-332.

[21] J. PUdykiewicz AND A. Staniforth, Some properties and comparative performance of the semi-Lagrangian method of robert in the solution of the advection-diffusion equation, Atmosphere-Ocean, 22 (1984), pp. 283-308.

[22] M. ReEves AND R. M. CRANwell, User's Manual for the Sandia Waste-Isolation Flow and Transport Model (SWIFT) Release 4.81, Sandia Report Nureg/CR-2324, SAND 81-2516, GR, Albuquerque, NM, 1981.

[23] T. F. Russell AND R. V. TRuJillo, Eulerian-Lagrangian localized adjoint methods with lvariable coefficients in multiple dimensions, Computational Methods in Surface Hydrology, G. Gambolati, A. Rinaldo, C. A. Brebbia, W. G. Gray, and G. F. Pinder, eds., Springer-Verlag, Berlin, 1990, pp. 357-363.

[24] C. SHU AND S. OsHER, Efficient implementation of essentially non-oscillatory shock capturing schemes, J. Comput. Phys., 77 (1988), pp. 439-471.

[25] P. K. SwEBY, High resolution schemes using flux limiters for hyperbolic conservation laws, SIAM J. Numer. Anal., 21 (1984), pp. 995-1011.

[26] V. Tноме́E, Galerkin Finite Element Methods for Parabolic Problems, Lecture Notes in Math. 1054, Springer-Verlag, New York, 1984.

[27] B. VAN LEER, On the relation between the upwind-differencing schemes of Godunov, EngquistOsher, and Roe, SIAM J. Sci. Stat. Comput., 5 (1984), pp. 1-20.

[28] E. VAROGLU AND W. D. L. Finn, Finite elements incorporating characteristics for onedimensional diffusion-convection equations, J. Comput. Phys., 34 (1980), pp. 371-389.

[29] H. WANG, A family of ELLAM schemes for advection-diffusion-reaction equations and their convergence analyses, Numer. Methods Partial Differential Equations, 14 (1998), pp. 739 780.

[30] H. Wang, M. Al-LaWatia, and S. A. TelyakovskiY, A Runge-Kutta characteristic method for first-order linear hyperbolic equations, Numer. Methods Partial Differential Equations, 13 (1997), pp. 617-661.

[31] H. Wang, H. K. Dahle, R. E. Ewing, M. S. Espedal, R. C. Sharpley, and S. Man, An ELLAM scheme for advection-diffusion equations in two dimensions, SIAM J. Sci. Comput., 20 (1999), pp. 2160-2194.

[32] H. WANG, R. E. Ewing, AND T. F. Russell, Eulerian-Lagrangian localized methods for convection-diffusion equations and their convergence analysis, IMA J. Numer. Anal., 15 (1995), pp. 405-459.

[33] J. J. Westerink And D. SheA, Consider higher degree Petrov-Galerkin methods for the solution of the transient convection-diffusion equation, Internat. J. Numer. Methods Engrg., 28 (1989), pp. 1077-1101.

[34] D. YANG, A characteristic-mixed method with dynamic finite element space for convectiondominated diffusion problems, J. Comput. Appl. Math., 43 (1992), pp. 343-353.

[35] Q. ZHU AND Q. Lin, Superconvergence Theory of FEM, Hunan Science Press, Changsha, China, 1989. 